\title{
TOPOLOGICAL OBSTRUCTIONS TO SMOOTHNESS FOR INFINITELY RENORMALIZABLE MAPS OF THE DISK
}

\author{
F. J. MOREIRA
}

\begin{abstract}
We analyze the signature type of a cascade of periodic orbits associated to period doubling renormalizable maps of the two dimensional disk. The signature is a sequence of rational numbers which describes how periodic orbits turn each other and is invariant by topological conjugacies that preserve orientation. We prove that in the class of area contracting maps the signature cannot be a monotone sequence. This explains why classical examples of infinitely renormalizable maps due to Bowen, Franks and Young cannot be achieved by smooth dissipative maps showing that there are topological obstructions to realize infinitely renormalizable maps in the area contracting case.
\end{abstract}

\section{INTRODUCTION}

In 1975, Bowen and Franks [2 gave the first example of a $C^{1}$ diffeomorphism of the sphere $S^{2}$ which is Kupka-Smale and possesses neither sinks nor sources. Using some more techniques Franks and Young 14 improved this result in 1980 and got a $C^{2}$ example. We will refer to these two examples as BFY models. Finally in 1989, a $C^{\infty}$ example was found by Gambaudo, Strien and Tresser [16] which we will refer as GST. It is still an open question wether there exists a real analytic example in $S^{2}$ with the mentioned properties.

The three cited examples are obtained by finding first a Kupka-Smale embedding of the two-disk with neither sinks nor sources, then glueing this embedding with its inverse to obtain the desired diffeomorphisms of $S^{2}$. It turns out that the embeddings of the two-disk are infinitely renormalizable by a period doubling cascade of disks surrounding a cascade of periodic points of saddle type.

Following [18, we can associate to a cascade of periodic orbits, a signature consisting of a sequence $\left(\ell_{n}\right)_{n \geq 0}$ of rational numbers such that each $\ell_{n}$ describes how the orbits of period $2^{n+1}$ are linked to the orbits of period $2^{n}$ (see next section for the details) and, is invariant by orientation preserving topological conjugacies. From the work of Gambaudo, Sullivan and Tresser, the signature is a convergent sequence for $C^{1}$ maps. By simple computation we easily derive in section 3 that the signature of the type BFY is a decreasing sequence converging to 0 .

The main result of this work deals with the obstruction to the realization of a monotone signature in the class of area contracting maps of the two dimensional disk. We prove (Theorem 3.1 and its Corollary 3.2) under mild assumptions on the geometry of the periodic cascade that, in the class of area contracting embeddings of the two disk

Date: October 31, 2018. 
(which contains GST models) monotone signatures cannot occur and thus, the decreasing sequence obtained in BFY model cannot be achieved for smooth dissipative maps.

The work is organized as follows. In section 2 we introduce the concept of signature and related properties. Then, in subection 2.1 we describe the properties of infinitely renormalizable maps needed to obtain the model types BFY and GST whose construction is sketched in the two subsequent subsections 2.2 and 2.3. Afterwards, section 2.4 is devoted to the bounded geometry property used throughout this work. The main result and its proof is established in section 3, where the contents of subsection 3.2 plays a crucial role, since therein we reduce our problem to the analysis of the signature of a multimodal endomorphism of the interval, and allows us to use one dimensional techniques stated in subsection 3.1.

\section{CASCAdes OF PERIOdiC ORBits}

Let $g$ be an orientation-preserving homeomorphism of the 2-disk $\mathbb{D}^{2}$. A cascade of periodic orbits of $g$ is a sequence of periodic orbits $\left\{O_{n}\right\}_{n \geq 0}$ with periods $\left\{q_{n}\right\}_{n \geq 0}$ such that, for each $n \geq 1$, we have:

(1) $q_{n}=a_{n} \cdot q_{n-1}$ with $q_{0}=1$ and $a_{n}>1$,

(2) there exists a collection of disjoint simple closed curves $C_{n}^{0}, \ldots C_{n}^{q_{n-1}-1}$, bounding the disjoint disks $D_{n}^{0}, \ldots D_{n}^{q_{n-1}-1}$, with the following properties:

(a) each $D_{n}^{i}$ contains one point of $O_{n-1}$ and $p_{n}$ points of $O_{n}$,

(b) $g\left(C_{n}^{i}\right)$ is isotopic to $C_{n}^{i+1 \bmod \left(q_{n-1}\right)}$ in the punctured disk $D^{2} \backslash \bigcup_{i \leq n} O_{i}$,

(c) $\bigcup_{0 \leq i \leq q_{n}-1} D_{n+1}^{i} \subset \bigcup_{0 \leq i \leq q_{n-1}-1} D_{n}^{i}$.

(d) The diameters of the disks $D_{n}^{i}$ go to zero with $n$.

Let $\left\{f_{t}\right\}_{t \in[0,1]}$ be an arc of embeddings joining the identity map $f_{0}$ to $g=f_{1}$, and $\left\{f_{t}\right\}_{t \in \mathbb{R}}$ be the extended arc of embeddings joining the identity to all iterates of $g$ defined by $g_{t}=f^{[t]} O f_{\{t\}}$ (where $[t]$ and $\{t\}$ denote the integer and decimal part of $t$, respectively). To each cascade of periodic orbits $\left\{O_{n}\right\}_{n \geq 0}$ we associate a signature $s\left(\left\{O_{n}\right\}_{n \geq 0}\right)=\left\{\lambda_{n}\right\}_{n \geq 1}$, where, for all $n \geq 1, \lambda_{n}$ is a rational number, $\lambda_{n}=l_{n}(f) / q_{n}$ and $l_{n}(f)$ is an integer defined as follows: In one of the $D_{n}^{i}$ 's, pick the point $x_{n-1}$ of $O_{n-1}$ and a point $x_{n}$ of $O_{n}$. Then, $l_{n}(f)$ is the algebraic number of loops that the vector

$$
\frac{f_{t}\left(x_{n}\right)-f_{t}\left(x_{n-1}\right)}{\left\|f_{t}\left(x_{n}\right)-f_{t}\left(x_{n-1}\right)\right\|}
$$

performs on the unit circle when $t$ goes from 0 to $q_{n}$ (here $\|$.$\| stands for the \mathbb{R}^{2}$ norm). Clearly, this number $l_{n}$ is independent of the choice of the $D_{n}^{i}$ and of the choice of the point $x_{n}$ in $D_{n}^{i}$.

Remark 2.1. Let $\left\{f_{t}^{\prime}\right\}_{t \in[0,1]}$ be another arc of homeomorphisms joining the identity map to $g$ and $\left\{f_{t}^{\prime}\right\}_{t \in \mathbb{R}}$ be the extended arc joining the identity map to all the iterates of $g$. We denote $l_{n}^{\prime}$ and $\lambda_{n}^{\prime}$ the quantities defined previously but computed for $\left\{f_{t}^{\prime}\right\}_{t \in[0,1]}$. Then there exists an integer $k$ such that $l_{n}^{\prime}=l_{n}+k q_{n}$ and thus $\lambda_{n}^{\prime}=\lambda_{n}+k$ for all $n \geq 1$. 
If the limit of the $\lambda_{n}$ 's exists when $n$ goes to infinity, we call it the asymptotic rotation number of the cascade $\left\{O_{n}\right\}_{n \geq 0}$ and denote it by $\omega\left(\left\{O_{n}\right\}_{n \geq 0}\right)$.

Let $\left\{O_{n}\right\}_{n \geq 0}$ be a cascade of periodic orbits of a map $f$ and $h$ an embedding of the 2-disk. The map $g=h^{-1} \circ f \circ h$ possesses also a cascade of periodic orbits $\left\{O_{n}^{\prime}\right\}_{n \geq 0}$ given by $O_{n}^{\prime}=h\left(O_{n}\right)$ for $n \geq 0$. An isotopy $\left\{f_{t}\right\}_{t \in[0,1]}$ between the identity and $f$ gives rise, in an natural way, to an isotopy $\left(g_{t}=h^{-1} \circ f_{t} \circ h\right)_{t \in[0,1]}$ between the identity and $g$. Computing $l_{n}(g)$ for this "conjugated" isotopy we have that

$$
l_{n}(g)=\left\{\begin{array}{rl}
l_{n}(f) & \text { if } h \text { preserves orientation } \\
-l_{n}(f) & \text { if } h \text { reverses orientation }
\end{array} .\right.
$$

Therefore, the signature of a cascade of periodic orbits is a topological invariant, that is to say it is invariant under conjugacy by an orientation-preserving homeomorphism of the two disk.

It is easy to check that, given a sequence of rational numbers $\left(\lambda_{n}\right)_{n>0}$, one can construct an orientation-preserving homeomorphism of the 2-disk with a cascade of periodic orbits $\left\{O_{n}\right\}_{n \geq 0}$ such that $s\left(\left\{O_{n}\right\}_{n \geq 0}\right)=\left\{\lambda_{n}\right\}_{n \geq 1}$. However there are topological obstructions to realize such a cascade for an orientation-preserving diffeomorphism of the 2-disk:

Theorem 2.2. [18] Any cascade of periodic orbits of a $C^{1}$ orientation-preserving diffeomorphism of the 2-disk possesses an asymptotic rotation number.

2.1. Infinitely renormalizable maps. Consider the unit disk $\mathbb{D}^{m}$ and a continuous map $g: \mathbb{D}^{m} \rightarrow \mathbb{D}^{m}$. Assume that there exists in $\mathbb{D}^{m}$ a topological disk $D$, that is to say, the image of $\mathbb{D}^{m}$ by a $C^{0}$-embedding $\xi(g): \mathbb{D}^{m} \rightarrow \mathbb{D}^{m}\left(\xi\left(\mathbb{D}^{m}\right)=D\right)$, such that there exists $p \geq 2$ satisfying:

(1) $D, g(D), \ldots g^{p-1}(D)$ have disjoint interiors,

(2) $g^{p}(D) \subset D$.

We call such a map renormalizable and the disk $D$ a domain of renormalization. In this setting it is natural to define the map

$$
\mathcal{R}(g)=\xi^{-1}(g) \circ g^{p} \circ \xi(g)
$$

which turns out to be the first return map in the disk $D$ rescaled to the disk $\mathbb{D}^{m}$. We call the map $\mathcal{R}(g)$ the renormalized map.

The functional operator $\mathcal{R}$, which associates a renormalized map to a renormalizable one, is called the renormalization operator.

If the renormalized map $\mathcal{R}(g)$ is again renormalizable we say that $g$ is twice renormalizable.It follows that we can define in a natural way $m$-times renormalizable maps and also infinitely renormalizable maps.

More precisely, a continuous map of the unit disk to itself is infinitely renormalizable if there exists a sequence $\mathbb{D}^{m} \supset D_{0} \supset D_{1} \supset \cdots \supset D_{n} \ldots$ of nested topological disks, and a sequence $\left(a_{n}\right)_{n \geq 0}$ of integers greater than or equal to 2 , such that, for each $n \geq 0$ :

(1) $D_{n}, g\left(D_{n}\right), \ldots g^{a_{o} \cdots a_{n}-1}\left(D_{n}\right)$ have disjoint interiors,

(2) $g^{a_{0} \cdot a_{1} \ldots a_{n}}\left(D_{n}\right) \subset D_{n}$. 
When more precision is required, we shall say that such a map is $\left(a_{n}\right)_{n \geq 0}$-infinitely renormalizable. We say that the nested sequence of disks $\left(D_{n}\right)_{n \geq 0}$ is a cascade for $g$ and the sets $f^{i}\left(\mathcal{D}_{n}\right)$, for $0 \leq i \leq a_{0} . a_{1} \ldots a_{n}-1$, are called the atoms of generation $n$ of $g$. Denoting by $\alpha_{k}(g): \mathbb{D}^{m} \rightarrow \mathbb{D}^{m}$ an embedding whose image is $D_{k}$ the $k$-renormalization of $g$ is defined as

$$
\mathcal{R}_{k}(g)=\alpha_{k}^{-1}(g) \circ g^{a_{0} \cdots a_{k-1}} \circ \alpha_{k}(g) .
$$

It is easy to check that the map $\mathcal{R}_{k}(g)$ is $\left(b_{n}\right)_{n \geq 0}$-infinitely renormalizable with $b_{n}=a_{n+k}$ for each $n \geq 0$ and the renormalized map of $\mathcal{R}_{k}(g)$ is the map $\mathcal{R}_{k+1}(g)$, under the natural scaling $\xi\left(\mathcal{R}_{k}(g)\right)=\alpha_{k}^{-1} \circ \alpha_{k+1}$, that is to say

$$
\mathcal{R}_{k+1}(g)=\xi^{-1}\left(\mathcal{R}_{k}(g)\right) \circ \mathcal{R}_{k}(g)^{a_{k}} \circ \xi\left(\mathcal{R}_{k}(g)\right)=\underbrace{\mathcal{R} \circ \cdots \circ \mathcal{R}}_{k}(g) .
$$

We say that an infinite renormalizable map is of bounded combinatorial type if the sequence $\left(a_{n}\right)_{n \geq 0}$ is bounded.

In the case of the interval $\mathbb{D}^{1}=[-1,1]$, it is natural to consider only the change of variables that are affine maps, since the image of any embedding $\alpha:[-1,1] \rightarrow[-1,1]$ is a nondegenerate interval, and thus we can carry $[-1,1]$ onto $\alpha([-1,1])$ by an affine map.

Let $\mathcal{U}$ be the set of real-analytic maps $g:[-1,1] \rightarrow[-1,1]$, satisfying

(1) $g$ is strictly increasing in $[-1,0]$ and strictly decreasing in $[0,1]$, that is to say, $g$ is unimodal with 0 as the critical point.

(2) $g(0)=1$ and $g^{\prime \prime}(0) \neq 0$.

(3) By setting $a=-g(1), b=g(a)$ we have

(a) $0<a<b<1$.

(b) $g(b)=g^{2}(a)<a$.

Any map $g \in \mathcal{U}$ maps $[-a, a]$ onto $[b, 1]$, and $[b, 1]$ onto $[-a, g(b)] \subset[-a, a]$. Moreover $g \circ g$ is again unimodal in $[-a, a]$. Then, in $\mathcal{U}$ we can define the renormalization operator $\mathcal{R}$ by

$$
\mathcal{R}(g)(x)=\frac{1}{g(1)} g^{2}(g(1) x) .
$$

In order to explain quantitative universal phenomena appearing in bifurcation in one parameter families of maps in the class $\mathcal{U}$, Coullet and Tresser ([9], 10]) and Feigenbaum [13, conjectured the following scenario for the structure of the renormalization operator defined above.

Proposition 2.3. The operator $\mathcal{R}$ is a bounded $C^{2}$ operator having a fixed point $\phi \in \mathcal{U}$ with the following properties:

(1) $\phi(x)=r\left(x^{2}\right)$, where $r$ is an analytic homeomorphism defined in a neighbourhood of $[-1,1]$ in $\mathbb{C}$. In particular $\phi$ is symmetric.

(2) The derivative $D \mathcal{R}(\phi)$ is a compact operator whose spectrum has a unique eigenvalue $\delta=4.6692 \ldots$ outside the unit disk and all the other eigenvalues have modulus less than 1. Let $\rho$ be the eigenvector corresponding to the eigenvalue $\delta$. This eigenvector is of the form 
$\rho(x)=v\left(x^{2}\right)$, where $v$ is an analytic homeomorphism defined in a neighbourhood of $[-1,1]$ in $\mathbb{C}$.

Lanford in 1982 21 gave the first complete proof of the above proposition. This proof is a rigorous analysis of the renormalization operator, based on numerical estimations made by computer. Later, in 1987 Eckman and Wittwer [12] gave a different proof of the same conjecture. Before that, the existence of fixed point for the renormalization without the characterization given in 2.3 had already be proved by Campanino and Epstein [5].

Using hyperbolic theory, the spectral property of the renormalization operator given in Proposition 2.3, gives locally, the following picture:

Proposition 2.4. In a neighbourhood of the fixed point $\Phi$ in the space $\mathcal{U}$, there exists an unstable manifold $W^{u}$ of dimension one tangent to an eigenvector $\rho:[-1,1] \rightarrow[-1,1]$ associated to de eigenvalue $\delta$ and a codimension one stable manifold $W^{s}$ where for every map $g \in W^{s}, \mathcal{R}_{n}(g)$ converges exponential fast to $\Phi$ as $n \rightarrow \infty$.

In 1992 Sullivan [24] gave a conceptual explanation of universality in renormalization, opening new directions in the Theory of Dynamical Systems.

Theorem 2.5. 24] There exists a space $\mathcal{E}$ of maps $g:[-1,1] \rightarrow[-1,1]$ of the form

$$
g=\psi_{1} \circ Q \circ \psi_{2}
$$

where $Q(x)=x^{2}, \psi_{1}, \psi_{2}:[-1,1] \rightarrow[-1,1]$ are orientation-preserving and orientationreversing respectively, with the following properties

(1) Every $f \in \mathcal{E}$ has an holomorphic extension $f^{\mathbb{C}}: W \rightarrow \mathbb{C}$ to a neighbourhood $W$ of $[-1,1]$ in $\mathbb{C}$.

(2) $\mathcal{E}$ has a strongly compact subset $\mathcal{C}$, that is to say, for every sequence $\left(f_{n}\right)_{n \geq 1}$ in $\mathcal{C}$, the holomorphic extension $\left(f_{n}^{\mathbb{C}}\right)_{n \geq 1}$ has an uniformly converging subsequence.

(3) For any map $f \in \mathcal{E}$ which is infinitely renormalizable and of the bounded combinatorial type there exists $n_{0}(f)$, such that $\mathcal{R}_{n}(f) \in \mathcal{C}$ for every $n \geq n_{0}$.

(4) $f, g \in \mathcal{C}$ have the same combinatorial type if and only if $\left(\mathcal{R}_{n}^{\mathbb{C}}(f)-\left(\mathcal{R}_{n}^{\mathbb{C}}(g)\right)_{n \geq 0}\right.$ converges uniformly to 0 .

(5) For any $C^{k+\text { Lip }}(k \geq 1)^{1}$ unimodal map $f:[-1,1] \rightarrow[-1,1]\left(a_{n}\right)_{n \geq 0}$-infinitely renormalizable with bounded combinatorial type, the $C^{1}$ distance of $\mathcal{R}_{n}(f)$ to $\mathcal{C}$ goes to 0 as $n$ goes to $\infty$. Moreover if $\left(a_{n}\right)_{n \geq 0}$ is periodic of period $N$, then $\left(\mathcal{R}_{n N}(f)\right)_{n \geq 0}(f)$ converges in the $C^{k}$-topology to a point $\phi_{\left(a_{n}\right)_{n \geq 0}} \in \mathcal{C}$ that is a periodic point of the renormalization operator, $\mathcal{R}_{N}\left(\phi_{\left(a_{n}\right)_{n \geq 0}}\right)=\phi_{\left(a_{n}\right)_{n \geq 0}}$.

The techniques introduced in the proof of this theorem are a beautiful combination of real and complex analysis. Let us finish this section pointing out a step in Sullivan's proof often referred to as "real bounds."

Theorem 2.6. 24] Let $f$ be an $C^{k+L i p}(k \geq 1)$ infinitely renormalizable unimodal map with combinatorial type bounded by $N$. Then, for all $n \geq 0$ :

\footnotetext{
${ }^{1}$ Actually $C^{k+Z y g m u n d}$. See for example 22
} 
(1) The renormalized maps $\mathcal{R}^{n}(f)$ have $C^{i}$-norm bounded by a constant $L_{f}(i)$ which depends only on $f$ and $i(1 \leq i \leq k)$. (The Lipschitz constant of the $k$ - derivative is also bounded by a constant depending only on $f$ )

(2) There exist two constants $a_{f}$ and $b_{f}$ which depend only on $f$ such that, if $\mathcal{I}$ is an atom of the generation $m$ and $\mathcal{J} \subset \mathcal{I}$ is an atom of the generation $m+1$ of $\mathcal{R}^{n}(f)$ then,

$$
0<a_{f} \leq \frac{|\mathcal{J}|}{|\mathcal{I}|} \leq b_{f}<1
$$

(where |.| stands for the diameter).

(3) All these bounds are "beau" (bounded and eventually universally (bounded)), that is to say, that for $n$ big enough, these bounds can be chosen so that they depend only on $N$.

In condition 2 the same estimate is true if $\mathcal{J}$ is a connected component of the complementary of the atoms of generation $m+1$ (a "gap") in $\mathcal{I}$. So for two atoms $\mathcal{J}, \mathcal{K} \subset \mathcal{I}$ of generation $m+1$ we also have $\operatorname{dist}(\mathcal{J}, \mathcal{K}) \geq a_{f}|\mathcal{I}|$. In dimension 2 , infinitely renormalizable maps are also frequently observed. For instance, they appear naturally in the infinitely dissipative situation for a map $(x, y) \mapsto(g(x), 0)$, where $g$ is an infinitely renormalisable map on the interval, and also in the area preserving case of a map exhibiting resonant islands.

In the sequel we give two examples of $(2)_{n \geq 0}$-infinitely renormalizable embedding of the two-disk.

2.2. The BFY Model. This construction was first introduced by R. Bowen and J. Franks [2] and provided a first example of $C^{1}$ Kupka-Smale diffeomorphism of the 2-sphere with no sinks nor sources. Later it was improved to a $C^{2}$ example by Franks and Young [14].

Consider an orientation-preserving $C^{\infty}$ diffeomorphism $g$ of the unit disk, which satisfies the following properties:

(1) $g$ is the identity in a thin annulus $A_{\epsilon}=\{x \in \mathbb{D},\|x\| \geq 1-\epsilon\}$;

(2) there exist four disjoint disks $D(0), D(1), D(2)$, and $D(3)$ of the same radius $\rho_{0}>\frac{1}{4}$ such that $g$ restricted to an $\epsilon$-neighborhood $D_{\epsilon}(i)$ of each disk $D(i)$ is a translation which maps the disk $D(i)$ exactly onto the disk $D(i+1 \bmod 4)$ and $g^{4}$ restricted to $D(0)$ is the identity.

(3) In the interior of $\mathbb{D} \backslash(D(0) \cup D(1) \cup D(2) \cup D(3)), g$ is a Morse-Smale system. Consider now an isotopy $G:[0,1] \times \mathbb{D} \rightarrow \mathbb{D}$ joining the identity, $i d=G(0, \cdot)$ to $G(1, \cdot)=g$ such that, for all $t \in[0,1], G(t, \cdot)$ restricted to $A_{\epsilon}$ is the identity and it maps rigidly the disks $D(i)$. For each positive integer $N$ and $0 \leq i \leq N-1$, by setting

$$
g(N, i)=G\left(\frac{i+1}{N}, \cdot\right) \circ G^{-1}\left(\frac{i}{N}, \cdot\right)
$$

we can write $g$ as the composition of $N$ maps $g(N, i)$,

$$
g=g(N, N-1) \circ \cdots \circ g(N, 1) \circ g(N, 0)
$$

that are $C^{r}$-close to the identity as $N$ goes to infinity,

$$
\|g(N, i)-i d\|_{r} \leq \frac{K(G, r)}{N} .
$$




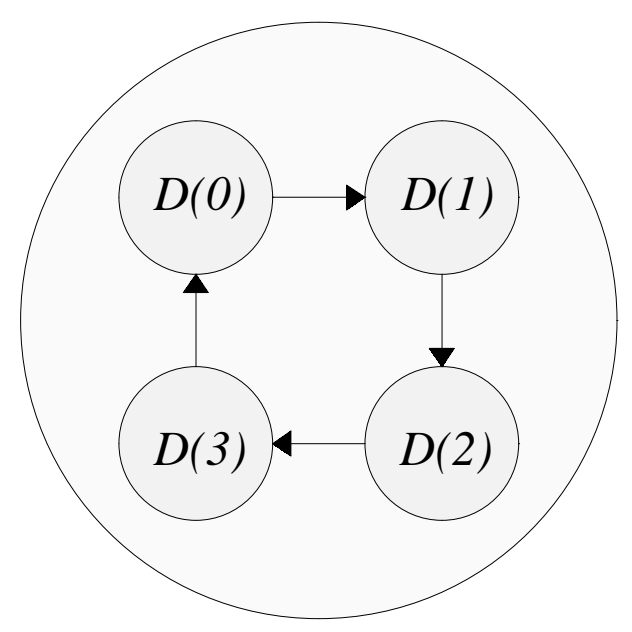

Figure 1. The map $g$

Let $\Lambda$ be an affine map that carries $\mathbb{D}$ to $D(0)$. Now we are going to construct a sequence of functions $\left(f_{n}\right)_{n \geq 0},\left(f_{0}=g\right),(4)_{1 \leq i \leq n+1}$-renormalizable with $\mathcal{R}_{i}\left(f_{n}\right)=f_{n-1}$ for $1 \leq i \leq n$. Moreover, $f_{n}$ has $4^{n+1}$ disks given by $D_{n}(i)=f_{n}^{i}\left(\Lambda^{n}(\mathbb{D})\right),\left(0 \leq i \leq 4^{n+1}-1\right)$, where $f_{n}$ is a rigid move and $f_{n}^{4^{n+1}}$ restricted to $D_{n}(0)$ is the identity (this means that we can define $\mathcal{R}_{n+1}\left(f_{n}\right)$ as the identity). The construction follows by induction. Define $f_{1}$ as

$f_{1}(x)=g(x)$ if $x$ is not in some $D(i),(0 \leq i \leq 3)$

$f_{1}(x)=g^{i+1} \circ \Lambda \circ g(4, i) \circ \Lambda^{-1} \circ g^{-i}(x)$ if $x$ is in some $D(i)$.

So, we have by the change of variables $\xi(f)=\Lambda, \mathcal{R}\left(f_{1}\right)=\Lambda^{-1} \circ f_{1}^{4} \circ \Lambda=g$. This function has the 16 disks with the properties needed for the first step of induction (recall that $G(t, \cdot)$ and thus, $g(k, i)$ rigidly permutes the four disks $D(0), D(1), D(2), D(3))$.

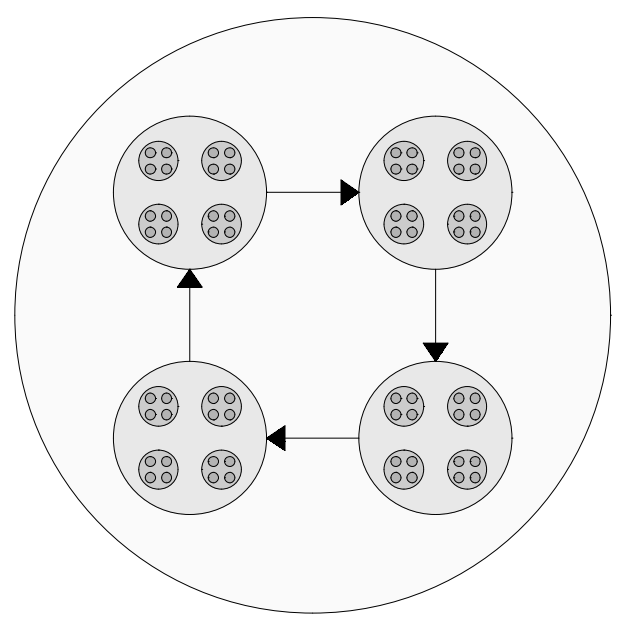

Figure 2. The atoms for the map $f_{2}$

Generally we define recursively $f_{n}$ by 
$f_{n}(x)=f_{n-1}(x)$ if $x$ is not in some $D_{n-1}(i),\left(0 \leq i \leq 4^{n}-1\right)$

$f_{n}(x)=f_{n-1}^{i+1} \circ \Lambda \circ g\left(4^{n}, i\right) \circ \Lambda^{-1} \circ f_{n-1}^{-i}(x)$ if $x$ is in some $D_{n-1}(i)$.

The map $f_{n}$ is again a $C^{\infty}$ diffeomorphism such that $\mathcal{R}\left(f_{n}\right)=f_{n-1}$ with $\xi\left(\mathcal{R}\left(f_{n}\right)\right)=\Lambda$. The annulus $A_{\epsilon}$ and its reduced copies allows the surgery which transforms $f_{n-1}$ into $f_{n}$ to be arbitrarily smooth.

Since $f_{n}$ differs from $f_{n-1}$ only in the disks $D_{n-1}(i)$, where $f_{n-1}$ is a rigid map, we have that

$$
\begin{aligned}
\left\|f_{n}-f_{n-1}\right\|_{r} & =\max _{i}\left\|\Lambda \circ g\left(4^{n}, i\right) \circ \Lambda^{-1}-i d\right\|_{r} \\
& \leq \max _{i}\left\|g\left(4^{n}, i\right)-i d\right\| \cdot \rho_{0}^{1-r} \\
& \leq K(G, r) \cdot \frac{\rho_{0}^{(1-r) n}}{4^{n}} .
\end{aligned}
$$

So, $f_{n}$ will be a converging sequence in the $C^{r}$ topology if we ensure that

$$
\sum_{n=1}^{\infty} K(G, r) \cdot \frac{\rho_{0}^{(1-r) n}}{4^{n}}<\infty
$$

Thus, by the choice $\rho_{0}>\frac{1}{4}$ this is true for $r \leq 2$. The $C^{2}$ limit sequence $f_{\infty}$ is $(4)_{n \geq 1^{-}}$ infinitely renormalizable by the scaling map $\Lambda$. Indeed this map is a fixed point of the renormalization operator $f \mapsto \Lambda^{-1} \circ f^{4} \circ \Lambda$ for the maps of $\mathbb{D}$ cyclically permuting the four disks $D(0), D(1), D(2), D(3)$. This map $f_{\infty}$ is $C^{2+\epsilon}$ but not $C^{3}$ (see [15]). The map $f_{\infty}^{2}$ obviously is a fixed point for the period doubling operator $f \mapsto \Lambda f^{2} \Lambda^{-1}$.

Starting with an map that rigidly permutes $a_{0}$ disks of radius $\rho_{0}>a_{0}^{-1}$ we can arrive, with this type of construction, to an $\left(a_{0}\right)_{n \geq 0}$-infinitely renormalizable map of the two disk. For any $a_{0}>2$ we can find in $\mathbb{D}^{2} a_{0}$ disks with radius $\rho_{0}>a_{0}$. However, there is no space in $\mathbb{D}^{2}$ for two disks with radius $\rho_{0}>\frac{1}{2}$. This is the reason why we construct an $(2)_{n \geq 0}$-infinitely renormalizable map in terms of an $(4)_{n \geq 0}$-infinitely renormalizable one.

The same kind of techniques described in this section apply to higher dimensions $m \geq 3$, yielding, as explained by Gambaudo et Tresser in [20], $C^{m+\epsilon}$ infinitely renormalizable maps on $\mathbb{D}^{m}$.

2.3. The GST model. Now we do a brief presentation of an example of real analytic embedding of the 2-disk which is infinitely renormalizable and yields a $C^{\infty}$ Kupka-Smale diffeomorphism of the 2-sphere with no sinks nor sources as described by J.M. Gambaudo, S. van Strien and C. Tresser [16]. Unlike the Bowen-Franks model, it does not result from a construction but from an exploration of the properties of the period doubling operator in one-dimension.

Let $\phi(x)=r\left(x^{2}\right)$ and $\rho(x)=v\left(x^{2}\right)$ be the maps given by Proposition 2.3. Consider a small neighbourhood of $[-1,1]$ in $\mathbb{R}^{2}$

$$
R(\Delta)=\left\{(x, y) \in \mathbb{R}^{2} \mid \exists x_{0} \in[-1,1] \text { with }\left|x-x_{0}\right|+|y|<\Delta\right\}
$$

and, the corresponding neighbourhood in $\mathbb{C}^{2}$

$$
C(\Delta)=\left\{(x, y) \in \mathbb{C}^{2} \mid \exists x_{0} \in \underset{8}{[-1,1]} \text { with }\left|x-x_{0}\right|+|y|<\Delta\right\} .
$$


Let $H(\Delta)$ be the set of bounded analytic maps $G: C(\Delta): \rightarrow \mathbb{C}^{2}$ such that $G(R(\Delta)) \subset \mathbb{R}^{2}$. Define the map $\Psi_{\alpha}$ by setting

$$
\Psi_{\alpha}(x, y)=\left(r\left(x^{2}-\alpha y\right), 0\right) .
$$

We know that $r$ is defined and is analytic in a neighbourhood of $[-1,1]$ in $\mathbb{C}$. Thus, for $\Delta$ small we have that the map $(r(x), 0)$ belongs to $H(\Delta)$. This implies, for $\Delta, \alpha>0$ sufficiently small that $\Psi_{\alpha} \in H(\Delta)$.

Since $\phi(x)=r\left(x^{2}\right)$ is a fixed point for the operator $\mathcal{R}(g)(x)=\lambda^{-1} g^{2}(\lambda x)(\lambda=\phi(1))$ for maps in $\mathcal{U}$, it is straightforward to verify that, by setting $\Lambda(x, y)=\left(\lambda x, \lambda^{2} y\right)$, the map $\Psi_{\alpha}$ is for $0<\alpha<1$ a fixed point for the operator $\mathcal{N}$ defined by:

$$
\mathcal{N}(g)=\Lambda^{-1} \circ g^{2} \circ \Lambda \text {. }
$$

Collet, Eckmann and Koch [8] have proved that $\mathcal{N}$ is in fact a $C^{2}$ bounded operator from a neighbourhood of $\Psi_{\alpha}$ in $H(\Delta)$ into $H(\Delta)$. In the same work it is proved that $D \mathcal{N}\left(\Psi_{\alpha}\right)$ is a compact operator from $H(\Delta)$ into itself. Although 1 is an eigenvalue of $D \mathcal{N}\left(\Psi_{\alpha}\right)$, in the cited work [8] it is introduced another operator $T_{\alpha}$ defined in a neighbourhood of $\Psi_{\alpha}$ in $H(\Delta)$ into $H(\Delta)$, for which $\Psi_{\alpha}$ is an hyperbolic fixed point. This operator $T_{\alpha}$ is a small perturbation of the operator $\mathcal{N}$. We summarize the properties of $T_{\alpha}$ in the next proposition.

Proposition 2.7. [8] For $\Delta, \alpha>0$ sufficiently small, there exists a $C^{2}$ transformation $T_{\alpha}$ from a neighbourhood of $\Psi_{\alpha}$ in $H(\Delta)$ into $H(\Delta)$ with the following properties.

(1) $T_{\alpha}\left(\Psi_{\alpha}\right)=\Psi_{\alpha}$.

(2) $T_{\alpha}(G)$ reads:

$$
T_{\alpha}(G)=\Lambda_{G}^{-1} \circ G^{2} \circ \Lambda_{G},
$$

where $\Lambda_{G}: C(\Delta) \rightarrow C(\Delta)$ is an embedding belonging to $H(\Delta)$ such that $\Lambda_{G} \rightarrow \Lambda$ as $G \rightarrow \Lambda$.

(3) The derivative $D T_{\alpha}\left(\Psi_{\alpha}\right)$ of $T_{\alpha}$ in $\Psi_{\alpha}$ is a compact operator whose spectrum has a unique eigenvalue $\delta=4.6692 \ldots$ outside the unit disk and all the other eigenvalues have modulus less than 1 . The eigenvector corresponding to this eigenvalue $\delta$ is of the form $\left(v\left(x^{2}-\alpha y\right), 0\right)$.

Let us set

$$
B_{G}(\Delta)=\Lambda_{G}(R(\Delta)) .
$$

Since $\Lambda_{G}$ sends $C(\Delta)$ into $C(\Delta)$ and $\Lambda_{G} \in H(\Delta)$ we have that $B_{G}(\Delta) \subset R(\Delta)$. Besides this, from the fact that for $\Delta>0$ small $\Phi([-1-\Delta, 1+\Delta]) \subset]-1-\Delta, 1+\Delta[$, we can easily derive (see [16] for further details) that, for $\Delta, \alpha>0$ sufficiently small and every $G \in H(\Delta)$ sufficiently close to $\Psi_{\alpha}$, one has that $G(R(\Delta)) \subset R(\Delta)$.

From the fact that $[\lambda,-\lambda] \cap \Phi([\lambda,-\lambda])=\emptyset$ and $\Phi^{2}[\lambda,-\lambda] \subset[\lambda,-\lambda]$, it is simple to derive that for $\Delta, \alpha>0$ we have that $B_{G}(\Delta)$ and $G\left(B_{G}(\Delta)\right)$ are disjoint and $G^{2}\left(B_{G}(\Delta)\right) \subset$ $B_{G}(\Delta)$. This means that in fact $T_{\alpha}(G)$ is a renormalized map of $G$.

Now, we know from Proposition 2.7, that $\Psi_{\alpha}$ is a hyperbolic fixed point of $T_{\alpha}$. Moreover, since $D T_{\alpha}$ has only one eigenvalue of modulus greater than one, the local stable manifold $\mathcal{W}_{l o c}^{s}\left(T_{\alpha}\right)$ of $T_{\alpha}$ in $H(\Delta)$ has codimension one. For every $G \in \mathcal{W}_{l o c}^{s}\left(T_{\alpha}\right)$ we have that $G$ is 

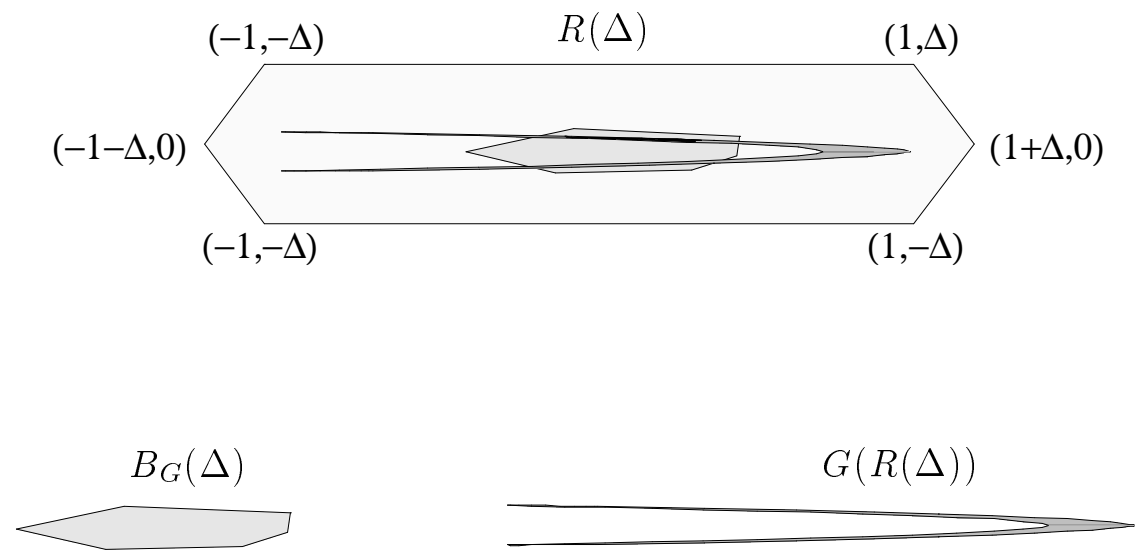

Figure 3. $G(R(\Delta))$ and $B_{G}(\Delta)$ for $G$ close to $\Psi_{\alpha}$.

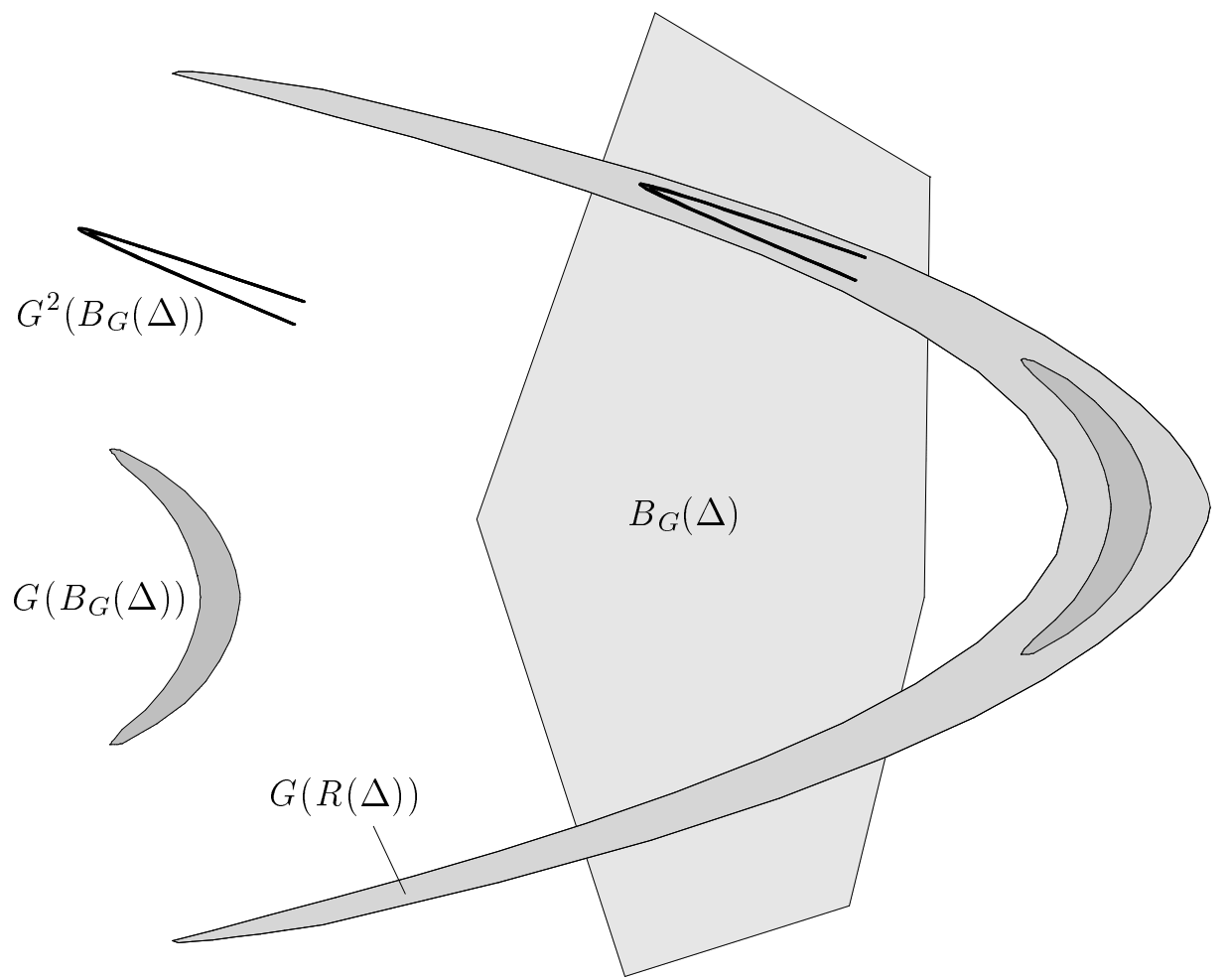

Figure 4. $B_{G}(\Delta), G\left(B_{G}(\Delta)\right)$ and $G^{2}\left(B_{G}(\Delta)\right)$ for $G$ close to $\Psi_{\alpha}$ (with a large zoom in the second coordinate).

infinitely renormalizable ${ }^{2}$ and its $n^{\text {th }}$ renormalized map is $T_{\alpha}^{n}(G)$. Consider a continuous

\footnotetext{
${ }^{2}$ More precisely we should say the map $g_{\mid R(\Delta)}: R(\Delta) \rightarrow R(\Delta)$ is infinitely renormalizable
} 
family of real analytic embeddings $\left(f_{\mu, \epsilon}\right)_{\mu \geq 0, \epsilon \geq 0}$ defined on $R(\Delta)$ satisfying the following conditions:

(1) $f_{0,0}=\Psi_{\alpha}$

(2) For every $\mu \geq 0$ and $\epsilon \geq 0\left(f_{\mu, \epsilon}\right)$ admits an extension in $H(\Delta)$.

(3) $(\mu, \epsilon) \neq\left(\mu^{\prime}, \epsilon^{\prime}\right)$ implies that $f_{\mu, \epsilon} \neq f_{\mu^{\prime}, \epsilon^{\prime}}$.

For example, we can define $f_{\mu, \epsilon}(x, y)$ as

$$
f_{\mu, \epsilon}(x, y)=\left(r\left(x^{2}-\alpha y\right)+\mu v\left(x^{2}-\alpha y\right), \epsilon x\right) .
$$

A family in these conditions will necessarily cross, for small parameters $\mu$ and $\epsilon$, the (codimension-one) local stable manifold $\mathcal{W}_{l o c}^{s}\left(T_{\alpha}\right)$. Thus we have embeddings $f_{\mu, \epsilon}$ infinitely renormalizable and their renormalizable maps $\mathcal{R}_{n}\left(f_{\mu, \epsilon}\right)=T_{\alpha}^{n}\left(f_{\mu, \epsilon}\right)$ converge to the degenerate map $\Psi_{\alpha}$ as $n \rightarrow \infty$.

2.4. Signature for maps with bounded geometry. The maps found in 2.2 and 2.3 have a good behaviour under renormalization. They satisfy the following definition.

Definition 2.8. We say that an infinitely renormalizable map $f$ of the $m$-disk, has $C^{k}$ bounded renormalization if it has bounded combinatorial type and there exists a sequence of constants $\left(M_{f}(i)\right)_{0 \leq i \leq k}$, where $M_{f}(i)$ depends only on $f$ and $i$ such that for all $n \geq 0$ :

(1) the renormalized maps $\mathcal{R}_{n}(f)$, the scaling maps $\xi_{n}(f)=\xi\left(\mathcal{R}_{n}(f)\right)$, and their inverse $\xi_{n}^{-1}$, are $C^{k+\epsilon}$ (in particular $f$ is $C^{k+\epsilon}$ ) and their $C^{i}$-norm for $1 \leq i \leq k$ are bounded by $M_{f}(i)$.

(2) $M_{f}(k)$ is also a $\epsilon$-Holder constant for $D^{k} \mathcal{R}_{n}(f), D^{k} \xi_{n}(f)$ and $D^{k} \xi_{n}^{-1}(f)$

Indeed in all the examples we have seen of infinitely renormalizable maps the scaling maps $\xi_{n}(f)$ are $C^{\infty}$. They also are convergent as $n \rightarrow \infty$, and so they have the "beau" bound required in the above definition for any $k$. The differentiability of the bounded renormalization is given in all examples by the differentiability of the renormalized maps. In the case of a $C^{k+\text { Lip }}$ infinitely renormalizable unimodal map of the interval, Theorem 2.6] states that it has $C^{k}$-bounded renormalization. Moreover if such a map is $C^{\infty}$ it has $C^{k}$ bounded renormalization for every $k$. The Bowen Franks model in Section has $C^{2}$-bounded renormalization. Section 2.3 gives us an infinitely renormalizable map of the two disk with $C^{k}$-bounded renormalization for every $k$.

Moreover all the atoms of this infinitely renormalizable maps have an universal bounded behaviour stated in the following definition.

Definition 2.9. We say that an infinitely renormalizable map $f$ of the $m$-disk, has $C^{k}$ bounded geometry if it has $C^{k}$-bounded renormalization and there exists constants $0<a_{f}<$ $b_{f}<1$ which depend only on $f$ such that for all $n \geq 0$, if $\mathcal{I}$ is an atom of the generation $m$ of $\mathcal{R}_{n}(f)$ and $\mathcal{J} \subset \mathcal{I}$ is an atom of the generation $m+1$, then

$$
a_{f} \leq \frac{|\mathcal{J}|}{|\mathcal{I}|} \leq b_{f}
$$


(where |.| stands for the diameter). For another atom $\mathcal{K} \subset \mathcal{I}$ of generation $m+1$ we also have

$$
a_{f} \leq \frac{\operatorname{dist}(\mathcal{J}, \mathcal{K})}{|\mathcal{I}|} \leq b_{f}
$$

Remark 2.10. Actually, the above relation in the ratios of the atoms forces $f$ to have bounded combinatorial type.

Remark 2.11. Bounded geometry implies that the diameter of an atom $I_{l}$ of generation $l$ goes to zero exponentially fast when $l$ increases. In fact, it is straightforward to derive inductively that,

$$
a_{f}^{l-1} \cdot\left|\mathbb{D}^{m}\right|<\left|I_{l}\right|<b_{f}^{l-1} \cdot\left|\mathbb{D}^{m}\right| .
$$

For simplicity we always say that a $C^{k}$ infinitely renormalizable map satisfies the bounded geometry hypothesis (respectively bounded renormalization hypothesis) if it satisfies the $C^{k}$-bounded geometry properties (respectively $C^{k}$ - bounded geometry renormalization). If $f$ is $C^{\infty}$ we say that it has bounded geometry (respectively bounded renormalization) if it satisfies the $C^{k}$-bounded geometry properties (respectively the $C^{k}$-bounded renormalization properties ) for every $k \geq 0$.

Remark: To have bounded geometry is a strong assumption. An infinitely renormalizable map of the interval $f \in \mathcal{U}^{1+L i p}$ with bounded combinatorial type, satisfies this assumption (Theorem [2.6), and recently it has been proved that this is also the case for other onedimensional maps with finitely many critical points (see [23]). However there is no result of this type for two-dimensional maps. If an $\left(a_{n}\right)_{n}$-infinitely renormalizable map $f$ has the property that any two atoms of the same generation are disjoint (we recall that in our definition they only have disjoint interiors), it follows, from Brouwer fixed point theorem, that the map $f$ possesses a sequence of periodic orbits $\left\{O_{n}\right\}_{n \geq 0}$ with periods $\left\{q_{n}\right\}_{n \geq 0}$ such that $q_{n}=a_{n} . q_{n-1}$ with $q_{0}=1$.

From now on we will consider an infinitely renormalizable map of the two-disk with the bounded geometry hypothesis. In particular, we know that under this hypothesis we have that the distance of two distinct atoms of the same generation is greater than 0 . Thus they are disjoint. The collection of disjoint simple closed curves $C_{n}^{0}, \ldots C_{n}^{q_{n-1}-1}$, bounding the disjoint disks $D_{n}^{0}, \ldots D_{n}^{q_{n-1}-1}$, where $D_{n}^{i}=f^{i}\left(\mathcal{D}_{n}(f)\right)$ satisfies:

(1) each $D_{n}^{i}$ contains one point of $O_{n-1}$ and $a_{n}$ points of $O_{n}$,

(2) $f\left(C_{n}^{i}\right)=C_{n}^{i+1 \bmod \left(q_{n-1}\right)}$

$$
\bigcup_{0 \leq i \leq q_{n}-1}^{i} D_{n+1}^{i} \subset \bigcup_{0 \leq i \leq q_{n-1}-1} D_{n}^{i}
$$

(4) the diameter of the disks $D_{n}$ is less than $b_{f}^{n-1}\left|\mathbb{D}^{2}\right|$. Thus, it goes to zero as $n$ goes to infinity.

Therefore, we have associated to an infinitely renormalizable map, a cascade of periodic orbits.

Remark 2.12. The cascade of periodic orbits associated to an infinitely renormalizable map is not necessarily unique. 
This cascade for $f$ gives rise to a cascade of periodic orbits for $\mathcal{R}(f)$, where $O_{n}(\mathcal{R}(f))=$ $\xi(f)^{-1} \circ f^{a_{0}} \circ \xi(f)\left(O_{n+1}\right)$.

Consider now the signature of $f,\left(\frac{l_{n}(f)}{a_{0} \cdots a_{n}}\right)_{n \geq 1}$ computed by an isotopy $\left\{f_{t}\right\}_{t \in[0,1]}$ of the disk joining the identity $f_{0}=i d$ to $f_{1}=f$. The image $f_{t}(\mathcal{D}(f))$ of the domain of renormalization $\mathcal{D}(f))=\xi(f)\left(\mathbb{D}^{2}\right)$ is a disk, with $f_{0}(\mathcal{D}(f))=\mathcal{D}(f)$ and $f_{a_{0}}(\mathcal{D}(f)) \subset \mathcal{D}(f)$. We can find a continuous arc $\left(A_{t}: \mathcal{D}(f) \rightarrow \mathbb{D}^{2}\right)_{t \in\left[0, a_{0}\right]}$ of affine maps, with $A_{0}=A_{a_{0}}=i d$ and $A_{t} \circ f_{t}(\mathcal{D}(f)) \subset \mathcal{D}_{0}(f)$. With this arc of affine maps, we can construct an isotopy $F_{t}=A_{t} \circ f_{t}$, $t \in\left[0, a_{0}\right]$ of $\mathcal{D}(f)$. For the computation of $l_{n+1}(f)$, we look for the number of loops that the vector

$$
\frac{f_{t}\left(x_{n+1}\right)-f_{t}\left(x_{n}\right)}{\left\|f_{t}\left(x_{n+1}\right)-f_{t}\left(x_{n}\right)\right\|}
$$

performs when $t$ goes from 0 to $a_{0} a_{1} \cdots a_{n}$. Denote by $l_{n+1}^{\prime}(f)$ the number of loops that the vector

$$
\frac{F_{t}\left(x_{n+1}\right)-F_{t}\left(x_{n}\right)}{\left\|F_{t}\left(x_{n+1}\right)-F_{t}\left(x_{n}\right)\right\|}
$$

performs when $t$ goes form 0 to $a_{0} a_{1} \cdots a_{n}$. Since $A_{t}$ are affine maps, we have that $l_{n+1}^{\prime}(f)=$ $l_{n+1}(f)$. Computing $l_{n}(\mathcal{R}(f))$ for the isotopy $\xi^{-1}(f) \circ F_{t} \circ \xi(f)$ we have, attending to 1 , that $l_{n}(\mathcal{R}(f))=l_{n+1}^{\prime}(f)$, if $\xi(f)$ preserves orientation and, $l_{n}(\mathcal{R}(f))=-l_{n+1}^{\prime}(f)$ if $\xi(f)$ reverses orientation. Thus we have

$$
l_{n}(\mathcal{R}(f))=\left\{\begin{aligned}
l_{n+1}(f) & \text { if } \xi(f) \text { preserves orientation } \\
-l_{n+1}(f) & \text { if } \xi(f) \text { reverses orientation. }
\end{aligned}\right.
$$

Of course in the above equalities we assume that the computation for $l_{n}(\mathcal{R}(f))$ is made using the isotopy $\xi^{-1}(f) \circ A_{t} \circ f_{t} \circ \xi(f)$. By setting $\alpha_{k}=\xi_{0}(f) \circ \cdots \circ \xi_{k-1}(f)$, the renormalized map $\mathcal{R}_{k}(f)$ has a cascade $\left(O_{n}\left(\mathcal{R}_{k}(f)\right)_{n \geq 1}\right.$ given by $O_{n}\left(\mathcal{R}_{k}(f)\right)=\alpha_{k}\left(O_{n+k}(f)\right)$. Inductively we have for these two cascades:

$$
l_{n}\left(\mathcal{R}_{k}(f)\right)=\left\{\begin{aligned}
l_{n+k}(f) & \text { if } \alpha_{k} \text { preserves orientation } \\
-l_{n+k}(f) & \text { if } \alpha_{k} \text { reverses orientation. }
\end{aligned}\right.
$$

The equality given in 3 allows us to compute immediately the signature for a fixed point of the renormalization operator under a fixed change of coordinates $\Lambda$. If for an integer $a \geq 2$ and an embedding $\Lambda$ of the two disk we have $\mathcal{R}(f)=\Lambda^{-1} \circ f^{a} \circ \Lambda=f$ then, for $n \geq 1$ we have that:

$$
l_{n}(f)=\left\{\begin{aligned}
l_{n+1}(f) & \text { if } \Lambda \text { preserves orientation } \\
-l_{n+1}(f) & \text { if } \Lambda \text { reverses orientation }
\end{aligned}\right.
$$

and thus,

$$
l_{n}(f)=\left\{\begin{array}{rl}
l_{1}(f) & \text { if } \Lambda \text { preserves orientation } \\
(-1)^{n-1} l_{1}(f) & \text { if } \Lambda \text { reverses orientation } \\
13
\end{array} .\right.
$$


If we always choose an isotopy for which $l_{1}=1$, we have for the Bowen-Franks model $(\Lambda$ preserves orientation):

$$
s\left(\left\{O_{n}\right\}_{n \geq 0}\right)=\left(\frac{1}{2^{n}}\right)_{n \geq 0}
$$

and for the period doubling model

$$
s\left(\left\{O_{n}\right\}_{n \geq 0}\right)=\left(\frac{(-1)^{n-1}}{2^{n}}\right)_{n \geq 0} .
$$

\section{MAin RESUlT}

The main result of this chapter says that for smooth infinitely renormalizable embeddings of the two disk, area contracting and satisfying the bounded geometry hypothesis, the signature cannot be a monotonic sequence.

Theorem 3.1. Let $f$ be an $\left(a_{n}\right)_{n}$-infinitely renormalizable embedding of the 2-disk with $a_{n}=2$ for all $n$, and $\left(\frac{\ell_{n}}{2^{n}}\right)_{n}$ the signature of the cascade of periodic orbits associated to $f$. Assume that $f$ is $C^{\infty}$, has bounded geometry and contracts the area. Then the sequence $\left(\frac{\ell_{n}}{2^{n}}\right)_{n}$ alternates, that is to say, for each $N>0$ we can always find $n_{1}$ and $n_{0}$ great or equal to $N$, such that

$$
\frac{\ell_{n_{0}}}{2^{n_{0}}}<\frac{\ell_{n_{0}+1}}{2^{n_{0}+1}} \quad \text { and } \quad \frac{\ell_{n_{1}}}{2^{n_{1}}}>\frac{\ell_{n_{1}+1}}{2^{n_{1}+1}}
$$

As a consequence we get

Corollary 3.2. The sequence $\left(\frac{1}{2^{n}}\right)_{n}$ cannot be the signature of an $(2)_{n}$-infinitely renormalizable area-contracting embedding with bounded geometry.

Roughly speaking, the Bowen-Franks model cannot be realized in the class of area-contracting embeddings with bounded geometry. The proof of Theorem 3.1 is given in the last section, but before we need to recall a nice property of multimodal maps.

All the remaining work is devoted to the proof of the Theorem above. First, in section 3.1 we recall the results of multimodal maps that we need to achieve the proof. In section 3.2 we derive the reduction of the dynamics of the initial map to the dynamics of a multimodal endomorphism of the interval that allows to conclude the proof in the last section.

3.1. Cascade for multimodal maps of the interval. Let $g$ be a $(2)_{n \geq 0}$ infinitely renormalizable multimodal map of the interval $I=\left[c_{0}, c_{q+1}\right]$, with critical points $c_{1}<\ldots<c_{q}$. Denote by $I_{k}$ the interval of monotonicity $\left[c_{k-1}, c_{k}\left[\right.\right.$. As usual $\left(\mathcal{D}_{n}(g)\right)_{n}$ denotes a cascade for $g$. We assume that $g$ satisfies the bounded geometry assumption, so that atoms of the same generation are disjoint and the diameter of the atoms of generation $n$ goes to zero as $n \rightarrow \infty$.

Under these conditions, in each atom $D_{n}^{i}=g^{i}\left(\mathcal{D}_{n}(g)\right)$, we have a periodic point $x_{n}$ of period $2^{n}$ and two periodic orbits $x_{1, n+1}, x_{2, n+1}$ of period $2^{n+1}$, with $x_{1, n+1}<x_{n}<x_{2, n+1}$. It is usual to call $x_{n}$ a father of $x_{i, n+1}(i=1,2)$. A sequence of periodic orbits $\left(O_{n}\right)_{n \geq 0}$, 
where for every $n$ the period of $O_{n}$ is $2^{n}$ and every point in $O_{n}$ has two sons in $O_{n+1}$, is called a period-doubling cascade of orbits for $f$.

For a sufficiently large generation the father and its sons belong to the same interval of monotonicity or are in two adjacent intervals $I_{k}, I_{k+1}$, and the father is always between two sons.

Using the fact that for any cascade of period doubling orbits each father is between two sons and, that globally we have the structure suggested by the Figure [5. Courcelle [11] was able to establish a useful relation for the number of periodic orbits in each interval of monotonicity:

Proposition 3.3. Let $\Phi(k, n)$ denote the number of periodic orbits of period $2^{n}$ of a period doubling cascade in the interval of monotonicity $I_{k}$ for $g$. For all $n \geq 0$ and for all $k \in\{0, \ldots, q\}$

$$
\Phi(k, n+1)=2 \Phi(k, n)+r(k, n)
$$

where for all $p_{2} \geq p_{1} \geq 0$,

$$
\left|\sum_{j=p_{1}}^{p_{2}} r(k, j)\right| \leq 2 .
$$

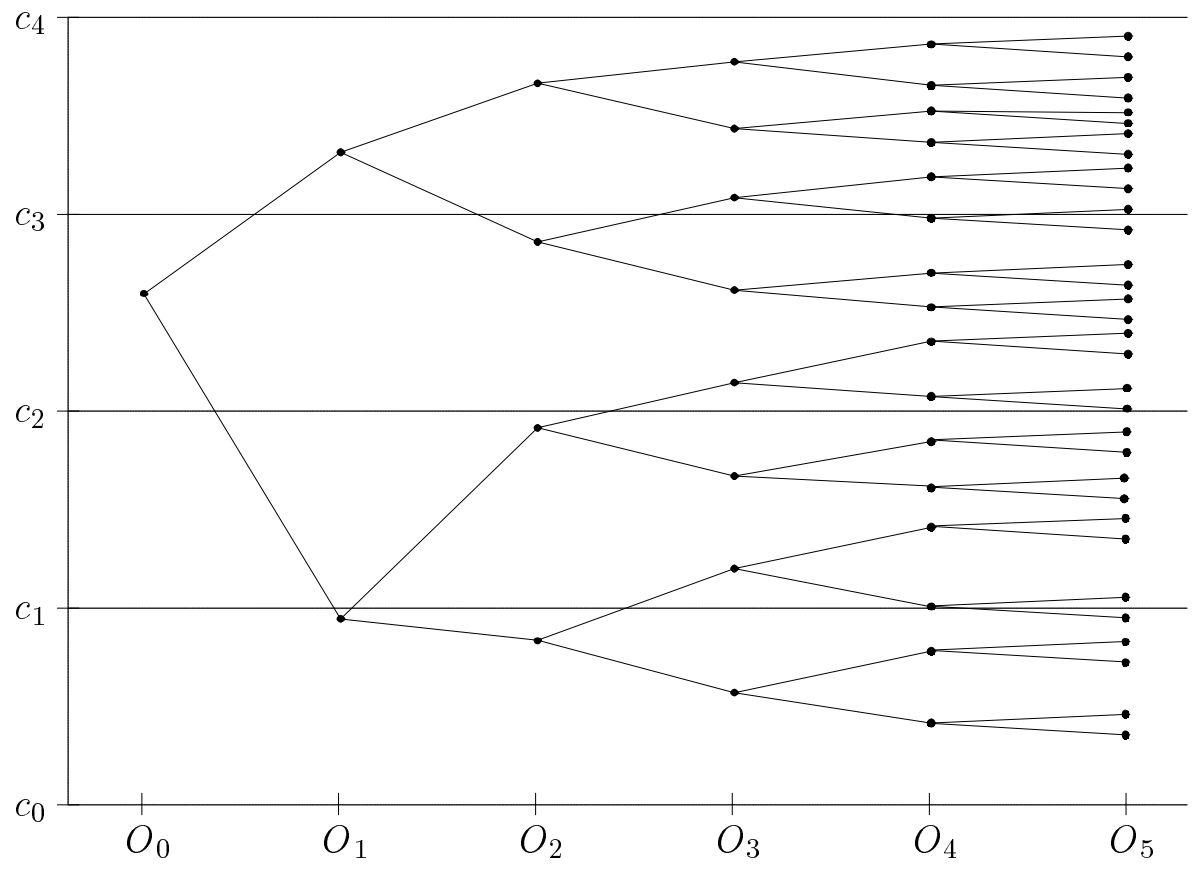

FiguRE 5. The structure of periodic orbits for multimodal maps. In this example, we have $\Phi(1,3)=1, \Phi(2,3)=2, \Phi(3,3)=2$ and $\Phi(4,3)=3$ 
3.2. Rigorous dynamics reduction. All along this section we assume that $f$ is an $\left(a_{n}\right)_{n \geq 0}$-infinitely renormalizable map of the two disk of the class $C^{k}$, where $k$ is greater or equal than 1 . We fix a cascade $\left(D_{n}\right)_{n \geq 1}$ of $f$ and its associated sequence of embeddings $\left(\alpha_{n}\right)_{n \geq 0}$ as defined in Section 1.1. So there is no ambiguity on the definition of the scaling functions $\xi_{n}(f)=\xi\left(\mathcal{R}_{n}(f)\right)=\alpha_{n-1}^{-1}(f) \circ \alpha_{n}(f)$. The $n$-renormalized of $f$ is

$$
\begin{aligned}
\mathcal{R}_{n}(f) & =\alpha_{n}^{-1}(f) \circ f^{a_{0} \cdots a_{n-1}} \circ \alpha_{n}(f) \\
& =\xi_{n-1}^{-1}(f) \circ \cdots \xi_{0}^{-1}(f) \circ f^{a_{0} \cdots a_{n-1}} \circ \xi_{0}(f) \cdots \xi_{n-1}(f) .
\end{aligned}
$$

Once the scaling functions fixed, there is no ambiguity on the definition of the renormalization operator $\mathcal{R}$ acting in the sequence of renormalized maps $\left(\mathcal{R}_{n}(f)\right)_{n \geq 0}$ :

$$
\mathcal{R}\left(\mathcal{R}_{n}(f)\right)=\xi_{n}^{-1} \circ \mathcal{R}_{n}^{a_{n}} \circ \xi_{n}=\mathcal{R}_{n+1}(f) .
$$

We will also assume that $f$ satisfies the bounded renormalization properties. We recall that under this assumption $f$ is of the bounded combinatorial type, that is to say $\left\{a_{n}, n \geq 0\right\}$ is a finite set, and thus, for every subsequence $\left(a_{\phi(n)}\right)_{n \geq 1}$, there is some $p_{0} \in\left\{a_{n}, n \geq 0\right\}$ with $a_{\phi(n)}=p_{0}$ for every $n \geq 1$. Moreover, the bounded renormalization hypothesis states that the sequence of renormalized maps $\left(\mathcal{R}_{n}(f)\right)_{n \geq 0}$ belongs to a space $\mathcal{U}^{k}=\mathcal{U}^{k}(f)$ defined by the $C^{k+\epsilon}$ maps $g: \mathbb{D}^{2} \mapsto \mathbb{D}^{2}$, satisfying:

(1) $\left\|D^{(i)} g\right\| \leq M_{f}(i)$ for every $1 \leq i \leq k$

(2) The Holder constant $\operatorname{Hold}\left(D^{(k)} g\right) \leq M_{f}(k)$.

Thus, for every sequence $\left(g_{n}\right)_{n}$ in $\mathcal{U}^{k}$, we have that for $i=0, \ldots, k,\left(D^{(i)} g_{n}\right)_{n}$, satisfies the equicontinuity property: $\forall \epsilon>0 \exists \delta>0$ such that for all $n \geq 0$

$$
\|x-y\|<\delta \Rightarrow\left\|D^{(i)} g_{n}(x)-D^{(i)} g_{n}(y)\right\|<\epsilon .
$$

From the Arzela-Ascoli Theorem there exists a $C^{k}$-converging subsequence of $\left(g_{n}\right)_{n}$. That is to say: there exists an increasing sequence of integers $(\phi(n))_{n \geq 1}$ and a $C^{k}$ map $g_{\infty}$ such that for every $0 \leq i \leq k$ the limit (in the $C^{k}$-topology) of the sequence $\left(D^{(i)}\left(g_{\phi(n)}\right)\right)_{n}$ exists and

$$
D^{(i)}\left(g_{\infty}\right)=\lim _{n \rightarrow \infty} D^{(i)}\left(g_{\phi(n)}\right) .
$$

By continuity, this map $g_{\infty}$ is also in $\mathcal{U}^{k}$. To summarize:

Theorem 3.4. (Arzela-Ascoli) For any sequence of maps $\left(g_{n}\right)_{n \geq 1}$ in $\mathcal{U}^{k}$, there exists a $C^{k}$-converging subsequence in $\mathcal{U}^{k}$.

Remark 3.5. This result implies that the limit of any $C^{0}$-converging sequence in $\mathcal{U}^{k}$ belongs to $\mathcal{U}^{k}$. In particular such $C^{0}$-limit is of class $C^{k}$.

When we refer to an accumulation point of a sequences in $\mathcal{U}^{k}$ we mean that it is an accumulation point in the $C^{0}$ topology. By the previous remark, these $C^{0}$-accumulation points are also in $\mathcal{U}^{k}$.

Corollary 3.6. The sequence of renormalized maps $\left(\mathcal{R}_{n}(f)\right)_{n}$ has an accumulation point $g_{\infty} \in \mathcal{U}^{k}$. 
Now let $g$ be an arbitrary accumulation point of the sequence $\left(\mathcal{R}_{n}(f)\right)_{n \geq 1}$ and $\phi(n)$ be a sequence such that $\left(\mathcal{R}_{\phi(n)}(f)\right)$ converges to $g$. From this sequence we can extract a subsequence $\psi(n)$ such that the sequence $\left(a_{\psi(n)}\right)_{n}$ is constant (say equal to $p_{0}$ ) and the sequence $\mathcal{R}_{\psi(n)+1}(f)=\mathcal{R}\left(\mathcal{R}_{\psi(n)}(f)\right)$ also converges in $\mathcal{U}^{k}$. Such a subsequence exists since $\left(a_{n}\right)_{n}$ takes only a finite number of values and $\mathcal{R}_{\phi(n)+1}(f)$ is a sequence in $\mathcal{U}^{k}$ (Arzela-Ascoli Theorem 3.4). Besides this, we also can impose (once more, by the Arzela-Ascoli theorem) that the corresponding scaling maps $\xi_{\psi(n)}(f)$ also converge. Since $(\psi(n))_{n}$ is a subsequence of $(\phi(n))_{n}$, we still have $g=\lim _{n \rightarrow \infty} \mathcal{R}_{\psi(n)}$.

Since for each $n$,

$$
\xi_{\psi(n)}(f) \circ \mathcal{R}_{\psi(n)+1}(f)=\mathcal{R}_{\psi(n)}^{p_{0}}(f) \circ \xi_{\psi(n)}(f),
$$

we obtain by continuity, taking limits, the relation

$$
\zeta_{0} \circ g=g^{p_{0}} \circ \zeta_{0},
$$

where

$$
\begin{aligned}
g & =\lim _{n \rightarrow \infty} \mathcal{R}_{\psi(n)+1}(f) \\
\zeta_{0} & =\lim _{n \rightarrow \infty} \xi_{\psi(n)}(f),
\end{aligned}
$$

From the bounded renormalization hypothesis, we also have that for $0 \leq i \leq k,\left\|D \xi_{n}^{-1}\right\| \leq M_{f}(i)^{3}$. This says that for every $n, \xi_{n}^{-1}$ is at least Lipschitz with Lipschitz constant smaller than $M_{f}(1)$, which means that:

$$
\left\|\xi_{n}^{-1}(f)\left(y_{1}\right)-\xi_{n}^{-1}(f)\left(y_{2}\right)\right\| \leq M_{f}(1) \cdot\left\|y_{1}-y_{2}\right\| \forall y_{1}, y_{2} \in \xi_{n}\left(\mathbb{D}^{2}\right) .
$$

This implies that for every $n$,

$$
\left\|\xi_{\psi(n)}(f)\left(x_{1}\right)-\xi_{\psi(n)}(f)\left(x_{2}\right)\right\| \geq \frac{1}{M_{f}(1)} \cdot\left\|x_{1}-x_{2}\right\| \forall x_{1}, x_{2} \in \mathbb{D}^{2}
$$

which leads, after taking limits as $n \rightarrow \infty$,

$$
\left\|\zeta_{0}\left(x_{1}\right)-\zeta_{0}\left(x_{2}\right)\right\| \geq \frac{1}{M_{f}(1)} \cdot\left\|x_{1}-x_{2}\right\| \forall x_{1}, x_{2} \in \mathbb{D}^{2} .
$$

From this last inequality it follows that $\zeta_{0}$ is a homeomorphism over its image and its inverse $\zeta_{0}^{-1}$ has the same differentiability as $\zeta_{0}$. From the fact that for each $n \geq 0$, we have:

$$
\mathcal{R}_{\psi(n)}^{p_{0}}(f)\left(\xi_{\psi(n)}(f)\left(\mathbb{D}^{2}\right)\right) \subset \xi_{\psi(n)}(f)\left(\mathbb{D}^{2}\right) .
$$

and, for $0 \leq i<j \leq p_{0}-1$

$$
\operatorname{Int}\left(\mathcal{R}_{\psi(n)}^{i}(f)\left(\xi_{\psi(n)}(f)\left(\mathbb{D}^{2}\right)\right)\right) \bigcap \operatorname{Int}\left(\mathcal{R}_{\psi(n)}^{j}(f)\left(\xi_{\psi(n)}(f)\left(\mathbb{D}^{2}\right)\right)\right)=\emptyset,
$$

we get, by continuity:

$$
g^{p_{0}}\left(\zeta_{0}\left(\mathbb{D}^{2}\right)\right) \subset \zeta_{0}\left(\mathbb{D}^{2}\right),
$$

\footnotetext{
${ }^{3}$ Notice that we cannot apply directly the Arzela-Ascoli Theorem to the sequence $\xi_{n}^{-1}$ since its domain of definition depends on $n$.
} 
and

$$
\operatorname{Int}\left(g^{i}\left(\zeta_{0}\left(\mathbb{D}^{2}\right)\right)\right) \bigcap \operatorname{Int}\left(g^{j}\left(\zeta_{0}\left(\mathbb{D}^{2}\right)\right)=\emptyset .\right.
$$

The above induced statements are summarized in the following lemma.

Lemma 3.7. For any accumulation point $g=\lim _{n \rightarrow \infty} \mathcal{R}_{\phi(n)}(f) \in \mathcal{U}^{k}$ there exists an accumulation point $p_{0}$ of the sequence $\left(a_{\phi(n)}\right)_{n}$, such that $g$ is renormalizable by $\mathcal{R}(g)=$ $\zeta_{0}^{-1} \circ g^{p_{0}} \circ \zeta_{0}$. Furthermore, $\mathcal{R}(g)$ is a limit point of the sequence $\left(\mathcal{R}_{\phi(n)+1}(f)\right)_{n}$ and $\zeta_{0}$ is an embedding which is an accumulation point of the sequence $\left(\xi_{\phi(n)}(f)\right)_{n}$.

Now we are going to apply this lemma inductively. From an arbitrary accumulation point $g$ of the sequence $\left(\mathcal{R}_{n}(f)\right)_{n}$, we obtain a renormalization $\mathcal{R}(g)=\zeta_{0}^{-1} \circ g^{p_{0}} \circ \zeta_{0}$ which is an accumulation point of the sequence $\left(\mathcal{R}_{\phi(n)+1}(f)\right)_{n}$. Suppose that we have defined $\mathcal{R}_{1}(g), \ldots, \mathcal{R}_{m}(g)$ with for $i=1, \ldots m$ :

$$
\begin{aligned}
\mathcal{R}_{i}(g) & =\zeta_{i-1}^{-1} \circ\left(\mathcal{R}_{i-1}(g)\right)^{p_{i-1}} \circ \zeta_{i-1} \\
& =\left(\prod_{t=0}^{i-1} \zeta_{t}^{-1}\right) \circ g^{p_{0} \cdots p_{i-1}} \circ\left(\prod_{t=0}^{i-1} \zeta_{t}\right)
\end{aligned}
$$

where $^{4}$

(1) $\zeta_{i}$ is an accumulation point of the sequence $\left(\xi_{\phi(n)+i-1}(f)\right)_{n}$.

(2) $\mathcal{R}_{i}(g)$ is a limit point of the sequence $\left(\mathcal{R}_{\phi(n)+i}(f)\right)_{n}$

(3) $p_{i-1}$ is an accumulation point of the sequence $\left(a_{\phi(n)+i-1}\right)$.

Then we can apply the previous lemma to the accumulation point $\mathcal{R}_{m}(g) \in \mathcal{U}^{k}$ and obtain that $\mathcal{R}_{m}(g)$ is renormalizable by

$$
\mathcal{R}_{m+1}(g)=\mathcal{R}\left(\mathcal{R}_{m}(g)\right)=\zeta_{m}^{-1} \circ\left(\mathcal{R}_{m}(g)\right)^{p_{m}} \circ \zeta_{m}
$$

where

(1) $\zeta_{m}$ is an accumulation point of the sequence $\left(\xi_{\phi(n)+m}\right)_{n}$,

(2) $\mathcal{R}_{m+1}(g)$ is a limit point of the sequence $\left(\mathcal{R}_{\phi(n)+m+1}\right)_{n}$,

(3) $p_{m}$ is an accumulation point of the sequence $\left(a_{\phi(n)+m}\right)_{n}$.

This yields:

Lemma 3.8. Any accumulation point $g=\lim _{n \rightarrow \infty} \mathcal{R}_{\phi(n)}(f)$ is $\left(p_{m}\right)_{m \geq 0}$-infinitely renormalizable with

$$
\begin{aligned}
\mathcal{R}_{m+1}(g) & =\zeta_{m}^{-1} \circ\left(\mathcal{R}_{m}(g)\right)^{p_{m}} \circ \zeta_{m} \\
& =\left(\prod_{i=0}^{m} \zeta_{i}^{-1}\right) \circ g^{p_{0} \cdots p_{m}} \circ\left(\prod_{i=0}^{k} \zeta_{i}\right)
\end{aligned}
$$

where for every $m \geq 0$ :

(1) $\zeta_{m}$ is an accumulation point of the sequence $\left(\xi_{\phi(n)+m}(f)\right)_{n}$,

(2) $\mathcal{R}_{m+1}(g)$ is a limit point of the sequence $\left(\mathcal{R}_{\phi(n)+m+1}(f)\right)_{n}$

(3) $p_{m}$ is an accumulation point of the sequence $\left(a_{\phi(n)+m}\right)_{n}$.

\footnotetext{
${ }^{4}$ Of course, we are doing an incorrect use of the symbol $\prod$ in order to simplify some notation.
} 
Remark 3.9. Let $g$ be an accumulation point of the renormalized maps $\mathcal{R}_{n}(f)$. All along this thesis, when we say that $g$ is $\left(p_{m}\right)_{m \geq 0}$-infinitely renormalizable or we refer to the renormalized maps $\mathcal{R}_{m}(g)$, we always assume that $\left(\mathcal{R}_{m}(g)\right)_{m}$, their corresponding scaling maps $\left(\zeta_{m}\right)_{m}$ and $\left(p_{m}\right)_{m}$ satisfy statements 1, 2 and 3 of the above lemma.

The renormalized maps $\mathcal{R}_{m}(g)$ and their corresponding scaling maps $\zeta_{m}$ are respectively, limit points of the sequence of renormalized maps $\left(\mathcal{R}_{n}(f)\right)_{n}$ and the scaling maps $\left(\xi_{n}(f)\right)_{n}$. Therefore, the renormalized maps $\mathcal{R}_{m}(g)$, the scaling maps $\zeta_{m}$, and their inverse $\zeta_{m}^{-1}$, are $C^{k+\epsilon}$ and their $C^{i}$-norm for $1 \leq i \leq k$ are bounded by $M_{f}(i)$. Moreover, $M_{f}(k)$ is also a $\epsilon$-Holder constant for $D^{k} \mathcal{R}_{m}(g), D^{k} \zeta_{m}$ and $D^{k} \zeta_{m}^{-1}(f)$. This is summarized in the next result.

Lemma 3.10. Any accumulation point $g$ of the sequence of renormalized maps $\mathcal{R}_{n}(f)$ satisfies the bounded renormalization properties.

The next lemma shows that, any accumulation point of the sequence $\mathcal{R}_{n}(f)$ can be seen as a renormalized map of another accumulation point of the sequence $\left(\mathcal{R}_{n}(f)\right)_{n}$.

Lemma 3.11. For any accumulation point $g=\lim _{n \rightarrow \infty} \mathcal{R}_{\phi(n)}(f)$ of the sequence of renormalized maps of $f$, there is another limit point $g_{-1}$ of the sequence $\mathcal{R}_{\Phi(n)-1}(f)$ such that $\mathcal{R}\left(g_{-1}\right)=g$.

Proof. We choose a subsequence $\psi_{1}(n)$ of $\phi(n)$, such that $\left(\mathcal{R}_{\psi_{1}(n)-1}(f)\right)_{n},\left(\xi_{\psi_{1}(n)-1}(f)\right)_{n}$ are convergent and $a_{\psi_{1}(n)-1}$ is constant. Let us define $g_{-1}=\lim _{n \rightarrow \infty} \mathcal{R}_{\psi_{1}(n)}(f), \zeta_{-1}=$ $\lim _{n \rightarrow \infty} \xi_{\psi_{1}(n)-1}(f)$ and $p_{-1}=a_{\psi_{1}(1)-1}$. Then we have

$$
\mathcal{R}\left(g_{-1}\right)=\zeta_{-1}^{-1} \circ g_{-1}^{p_{-1}} \circ \zeta_{-1}=g .
$$

This lemma is the first step of an inductive process. For an arbitrary limit point $g$ of the sequence $\mathcal{R}_{n}(f)$ it gives another accumulation point $g_{-1}$ of the sequence $\mathcal{R}_{n}(f)$ such that $\mathcal{R}\left(g_{-1}\right)=g$. For this $g_{-1}$ it gives an $g_{-2}$ such that $\mathcal{R}\left(g_{-2}\right)=g_{-1}$, and thus $\mathcal{R}_{2}\left(g_{-2}\right)=g$, In this way we obtain the following result:

Lemma 3.12. For any accumulation point $g$ of the sequence of renormalized maps $\mathcal{R}_{n}(f)$, there is a sequence of accumulation points $\left(g_{-\ell}\right)_{\ell \geq 1}$ of the same sequence $\mathcal{R}_{n}(f)$, such that

$$
\mathcal{R}_{\ell}\left(g_{-\ell}\right)=g .
$$

From now on, we will assume that $f$ satisfies the bounded geometry hypothesis. This means that in addition to the bounded renormalization properties, there exists constants $0<a_{f}<b_{f}<1$ which depend only on $f$ such that for all $n \geq 0$, if $\mathcal{I}$ is an atom of the generation $l-1$ of $\mathcal{R}_{n}(f)$ and $\mathcal{J}, \mathcal{K}$ are atoms of the generation $l$ of $\mathcal{R}_{n}(f)$ with $\mathcal{J}, \mathcal{K} \subset \mathcal{I}$, then

$$
a_{f} \leq \frac{|\mathcal{J}|}{|\mathcal{I}|} \leq b_{f} \quad \text { and } \quad a_{f} \leq \frac{\operatorname{dist}(\mathcal{J}, \mathcal{K})}{|\mathcal{I}|} \leq b_{f} .
$$

The next result states that accumulation points of the sequence $\mathcal{R}_{n}(f)$ also possess the bounded geometry property. 
Lemma 3.13. Any accumulation point $g_{\infty}$ of the sequence $\mathcal{R}_{n}(f)$ is an infinitely renormalizable map satisfying the bounded geometry properties. We can choose the bounds so that $a_{g}=a_{f}$ and $b_{g}=b_{f}$.

Proof. From Lemma 3.10 we know that $g_{\infty}$ satisfies the bounded renormalization properties. It remains to prove that given any atoms $\mathcal{I}, \mathcal{J}, \mathcal{K}$ of $\mathcal{R}_{m}\left(g_{\infty}\right)$, with $\mathcal{J}$ and $\mathcal{K}$ of generation $l, \mathcal{I}$ of generation $l-1$ and $\mathcal{J}, K \subset I$. statement 6 holds.

However, since for every $m \geq 0$ the maps $\mathcal{R}_{m}\left(g_{\infty}\right)$ are also accumulation points of $\left(\mathcal{R}_{\phi(n)}\right)_{n}$ we only need to prove that any arbitrary accumulation point $g$ of the sequence $\mathcal{R}_{n}(f)$ satisfies statement [6 for any atoms $\mathcal{I}, \mathcal{J}, \mathcal{K}$ of $g$, with $\mathcal{J}$ and $\mathcal{K}$ of generation $l, \mathcal{I}$ of generation $l-1$ and $\mathcal{J}, K \subset I$.

By definition,

$$
\begin{aligned}
\mathcal{I} & =g^{q(\mathcal{I})} \circ \zeta_{0}(g) \circ \cdots \circ \zeta_{l-1}(g)\left(\mathbb{D}^{2}\right) \\
\mathcal{J} & =g^{q(\mathcal{J})} \circ \zeta_{0}(g) \circ \cdots \circ \zeta_{l-2}(g)\left(\mathbb{D}^{2}\right) \\
\mathcal{K} & =g^{q(\mathcal{K})} \circ \zeta_{0}(g) \circ \cdots \circ \zeta_{l-2}(g)\left(\mathbb{D}^{2}\right)
\end{aligned}
$$

for integers $q(\mathcal{I}), q(\mathcal{J})$ and $q(\mathcal{K})$ satisfying

$$
\begin{aligned}
0 \leq q(\mathcal{I}) & \leq p_{0}(g) \cdots p_{l-1}(g)-1 \\
0 \leq q(\mathcal{J}) & \leq p_{0}(g) \cdots p_{l-2}(g)-1 \\
0 \leq q(\mathcal{K}) & \leq p_{0}(g) \cdots p_{l-2}(g)-1
\end{aligned}
$$

Notice that the condition $\mathcal{J}, \mathcal{K} \subset \mathcal{I}$ is the same as $q(\mathcal{J})$ and $q(\mathcal{K})$ being multiples of $q(\mathcal{I})$. Let us define,

$$
\begin{aligned}
\mathcal{I}_{n} & =\mathcal{R}_{\psi(n)}^{q(\mathcal{I})}(f) \circ \xi_{\phi(n)}(f) \circ \cdots \circ \xi_{\psi(n)+l-2}(f)\left(\mathbb{D}^{2}\right) \\
\mathcal{J}_{n} & =\mathcal{R}_{\psi(n)}^{q(\mathcal{J})}(f) \circ \xi_{\phi(n)}(f) \circ \cdots \circ \xi_{\psi(n)+l-2}(f)\left(\mathbb{D}^{2}\right) \\
\mathcal{K}_{n} & =\mathcal{R}_{\psi(n)}^{q(\mathcal{K})}(f) \circ \xi_{\phi(n)}(f) \circ \cdots \circ \xi_{\psi(n)+l-2}(f)\left(\mathbb{D}^{2}\right)
\end{aligned}
$$

where $\psi(n)$ is chosen so that

$$
\lim _{n \rightarrow \infty} \mathcal{R}_{\psi(n)}(f)=g
$$

and

$$
\lim _{n \rightarrow \infty} \xi_{\psi(n)+i}(f)=\zeta_{i}(g) \text { for } 0 \leq i \leq l-1 .
$$

For each $n, \mathcal{I}_{n}, \mathcal{J}_{n}$ and $\mathcal{K}_{n}$ are atoms of $\mathcal{R}_{\psi(n)}(f)$. We have that $\mathcal{J}_{n}$ and $\mathcal{K}_{n}$ are atoms of generation $l$ and $\mathcal{I}_{n}$ is of generation $l-1$. Moreover, since $q(\mathcal{I})$ divides $q(\mathcal{J})$ and $q(\mathcal{K})$ we have that $\mathcal{J}_{n}, \mathcal{K}_{n} \subset \mathcal{I}_{n}$.

From the bounded geometry of $f$ it follows that

$$
a_{f} \leq \frac{\left|\mathcal{J}_{n}\right|}{\left|\mathcal{I}_{n}\right|} \leq b_{f} \text { and } a_{f} \leq \frac{\operatorname{dist}\left(\mathcal{J}_{n}, \mathcal{K}_{n}\right)}{\left|\mathcal{I}_{n}\right|} \leq b_{f}
$$


Since, for any pair of subsets $A, B \subset \mathbb{D}^{2}$, the maps

$$
\begin{aligned}
\mathcal{C}^{0}\left(\mathbb{D}^{2}\right) & \longrightarrow \mathbb{R}^{+} \\
h & \longmapsto
\end{aligned}
$$

and

$$
\begin{aligned}
& \mathcal{C}^{0}\left(\mathbb{D}^{2}\right) \times \mathcal{C}^{0}\left(\mathbb{D}^{2}\right) \quad \longrightarrow \mathbb{R}^{+} \\
& \left(h_{1}, h_{2}\right) \quad \longmapsto \operatorname{dist}\left(h_{1}(A), h_{2}(B)\right)
\end{aligned}
$$

are continuous we get that

$$
\begin{aligned}
|\mathcal{I}| & =\lim _{n \rightarrow \infty}\left|\mathcal{I}_{n}\right| \\
|\mathcal{J}| & =\lim _{n \rightarrow \infty}\left|\mathcal{J}_{n}\right| \\
|\mathcal{K}| & =\lim _{n \rightarrow \infty}\left|\mathcal{K}_{n}\right| \\
\operatorname{dist}(\mathcal{J}, \mathcal{K}) & =\lim _{n \rightarrow \infty} \operatorname{dist}\left(\mathcal{J}_{n}, \mathcal{K}_{n} \mid\right) .
\end{aligned}
$$

Therefore these last four equalities together with statement $\mathbf{7}$ gives immediately that $\mathcal{I}$, $\mathcal{J}, \mathcal{K}$ satisfy condition 6 and thus, the result is proved.

From now on we assume that, beyond the bounded geometry hypothesis, the map $f$ contracts uniformly the area, i.e. there exists $b$ such that $|\operatorname{det}(D f(x))| \leq b<1$ for all $x$ in $\mathbb{D}^{2}$. Then we have the following result:

Lemma 3.14. Any accumulation point $g$ of the sequence $\mathcal{R}_{n}(f)$ is a singular map, i.e. $\operatorname{det}(D g(x))=0$ for all $x \in \mathbb{D}^{2}$.

Proof. We have

$$
\mathcal{R}_{n}(f)=\xi_{n-1}^{-1}(f) \circ \cdots \circ \xi_{0}^{-1}(f) \circ f^{a_{0} \cdots a_{n-1}} \circ \xi_{0}(f) \circ \cdots \circ \xi_{n-1}(f) .
$$

Since for any linear map $A$ in dimension two, we have $|\operatorname{det} A| \leq\|A\|^{2}$, it follows that the modulus of the determinant of the scaling functions $\xi_{i}(f)$ and its inverse are bounded by $M_{f}^{2}(1)$, and thus:

$$
\left|\operatorname{det} D \mathcal{R}^{n}(f)(x)\right| \leq M_{f}^{4 n}(1) \cdot b^{a_{0} \cdots a_{n-1}} \leq M_{f}^{4 n}(1) \cdot b^{2^{n}},
$$

and this quantity goes to 0 when $n$ goes to $\infty$. Thus, by continuity, any accumulation point of the sequence $\left(\mathcal{R}_{n}(f)\right)_{n \geq 0}$ is a singular map.

Despite the fact that an accumulation point of the sequence of renormalized maps $\mathcal{R}_{n}(f)$ is a singular map, the next lemma shows us that this map is not degenerate.

Lemma 3.15. Let $g$ be a $C^{1}$ infinitely renormalizable map of the 2-disk. For each $l \geq 0$, there exists an atom $J_{l}$, of the $l^{\text {th }}$ generation of $g$ and a point $x_{l}$ in $J_{l}$ such that $\left\|D g\left(x_{l}\right)\right\| \geq$ 1 .

Proof. We know that

$$
g^{p_{0}(g) \cdots p_{l-1}(g)}\left(\mathcal{D}_{l}(g)\right) \subset \mathcal{D}_{l}(g),
$$

where

$$
\mathcal{D}_{l}(g)=\xi_{0}(g) \ldots \xi_{l-1}(g)\left(\mathbb{D}^{2}\right)
$$


and that $g^{p_{0}(g) \cdots p_{l-1}(g)}$ permutes in $\mathcal{D}_{l}(g)$ the $p_{l}$ atoms of generation $l+1$ that belong to $\mathcal{D}_{l}(g)$. It follows that there exists a point $y_{l}$ in $\mathcal{D}_{l}(g)$ such that

$$
\left\|D g^{p_{0}(g) \cdots p_{l-1}(g)}\left(y_{l}\right)\right\| \geq 1 \text {. }
$$

Consequently, in one of the $p_{0}(g) \cdots p_{l-1}(g)-1$ first images of $\mathcal{D}_{l}(g)$, that is to say, in an atom $J_{l}$ of the $l^{\text {th }}$ generation of $g$, there is a point $x_{l}$, image of $y_{l}$ by some iterate of $f$, such that $\left\|D g\left(x_{l}\right)\right\| \geq 1$.

Let $g$ be an arbitrary accumulation point of the sequence of renormalized maps $\mathcal{R}_{n}(f)$. By Lemma 3.8, $g$ is $\left(p_{m}\right)_{m \geq 0}$-infinitely renormalizable and then Lemma 3.15 gives a sequence of points $\left(x_{l}\right)_{l \geq 0}$ such that for every $l, x_{l}$ belongs to an atom $J_{l}$ of the $l^{\text {th }}$ generation and $\left\|D g\left(x_{l}\right)\right\| \geq 1$. We can extract from it, a subsequence $\left(x_{\theta(l)}\right)_{l}$ which converges to some $x \in \mathbb{D}^{2}$. By continuity, we get $\|D g(x)\| \geq 1$. It follows that there exists an open neighbourhood $\mathcal{V}_{x}$ of $x$ such that, for all $y \in \mathcal{V}_{x},\|D g(y)\| \geq 1 / 2$. Thanks to Lemma 3.14, we know that the map $g$ is singular. Therefore, for all $y \in \mathcal{V}_{x}$, the dimension of the kernel of $D g$ is 1 . Let us recall the following trivialization theorem.

Theorem 3.16. let $U, V$ be open sets of $\mathbb{R}^{n}, r$ an integer $\leq n$ and $F: U \rightarrow V$ a $C^{k}$ $(1 \leq k \leq \infty)$ function such that $\operatorname{dim}(\operatorname{ker} D F(y))=r$ for every $y \in U$. Then for every $x \in U$ there exist two $C^{k}$ embeddings

$$
u_{x, F}:[-1,1]^{n} \rightarrow U \text { and } \quad v_{x, F}:[-1,1]^{n} \rightarrow V
$$

such that

$$
u_{x, F}(0, \ldots, 0)=x \text { and } v_{x, F}(0, \ldots, 0)=F(x)
$$

and, denoting the points of $[-1,1]^{n}$ by $\left(t_{1}, t_{2}\right) \in[-1,1]^{r} \times[-1,1]^{n-r}$ we have that

$$
\begin{aligned}
v_{x, F}^{-1} \circ F \circ u_{x, F}:[-1,1]^{r} \times[-1,1]^{n-r} & \longrightarrow \mathbb{R}^{n} \\
\left(t_{1}, t_{2}\right) & \longmapsto\left(0, t_{2}\right) .
\end{aligned}
$$

Remark 3.17. The maps $u=u_{x, F}, v=v_{x, F}$ in the above theorem depend continuously on $F$ and $x$. In particular, the continuity with respect to $F$, ensures that if $\left(F_{m}\right)_{m}$ is a $C^{1}$-converging sequence of functions satisfying the hypothesis of the theorem, then

$$
\bigcap_{m \geq 0} u_{x, F_{m}}\left([-1,1]^{n}\right)
$$

contains for some $\epsilon>0$ the ball $B_{\epsilon}(x)$.

Remark 3.18. For a map $F$ satisfying the hypothesis of Theorem 3.16 we have a $C^{k}$ foliation given by $u_{x, F}\left([-1,1]^{r} \times\{y\}\right)$, for $y \in[-1,1]^{n-r}$. Since $v_{x, F}^{-1} \circ F \circ u_{x, F}\left(t_{1}, t_{2}\right)=\left(0, t_{2}\right)$ it follows that $F \circ u\left(t_{1}, t_{2}\right)=v\left(0, t_{2}\right)=F \circ u\left(t_{1}, t_{2}\right) \forall-1 \leq t_{1} \leq 1$. This means that $F$ maps the leaves of the referred foliation into a point. These leaves are locally the leaves of the foliation given by the field of directions defined by ker DF.

We can apply Theorem 3.16 to the map $g$. In this way, we obtain for the point $x \in V_{x}$, a change of coordinates $u_{x, g}:[-1,1]^{2} \rightarrow V_{x}$ and $v_{x, g}:[-1,1]^{2} \rightarrow \mathbb{R}^{2}$ such that $u_{x, g}(0,0)=x$ and for every $y_{1}, y_{2} \in[-1,1]$ :

$$
g \circ u_{x, g}\left(y_{1}, y_{2}\right)=v_{x, g}\left(0, y_{2}\right) .
$$


This yelds that for every integer $m$ and $y_{1}, y_{2} \in[-1,1]$,

$$
g^{m} \circ u_{x, g}\left(y_{1}, y_{2}\right)=g^{m} \circ u_{x, g}\left(0, y_{2}\right) .
$$

From the fact that $u_{x, g}$ is an embedding mapping $\left.(0,0) \in\right]-1,1[2$ into $x$ we have that the point $x$ is in the interior of $u_{x, g}\left([-1,1]^{2}\right)$. Since $x=\lim _{l \rightarrow \infty} x_{\theta(l)}, x_{\theta(l)}$ will be in the interior of $u_{x, g}\left([-1,1]^{2}\right)$ for $l$ large. Recall now that $g$ satisfies the bounded geometry properties (Lemma 3.13) and then, the diameter of the atoms of the $l^{\text {th }}$ generation goes to 0 as $l$ goes to $\infty$. Thus, there exists $l_{0}$ such that for all $l \geq l_{0}, u_{x, g}\left([-1,1]^{2}\right)$ contains $J_{\theta(l)}$ (the atom of the $\theta(l)^{t h}$ generation of $g$ containing $\left.x_{\theta(l)}\right)$. The map $g$ sends every atom of the $\theta(l)^{t h}$ generation into itself after $p_{0}(g) \cdots p_{\theta(l)}(g)$ iterations (we are using notation given by Lemma 3.8):

$$
g^{p_{0}(g) \cdots p_{\theta(l)}(g)}\left(J_{\theta(l)}\right) \quad \subset \quad J_{\theta(l)} .
$$

By setting $I$ as the projection in the second coordinate of $u_{x, g}^{-1}\left(J_{\theta(l)}\right)$, statement 9 allows us to consider the map $g_{\theta(l)}=u_{x, g}^{-1} \circ g^{p_{0}(g) \cdots p_{\theta(l)}(g)} \circ u_{x, g}:[-1,1] \times I \rightarrow[-1,1] \times I$. By $8 g_{\theta(l)}$ reads:

$$
g_{\theta(l)}\left(x_{1}, x_{2}\right)=\left(g_{1, \theta(l)}\left(0, x_{2}\right), g_{2, \theta(l)}\left(0, x_{2}\right)\right) .
$$

Notice that $I$ is a non-degenerate interval since $J_{\theta(l)}$ contains atoms of generation $p_{0}(g) \cdots p_{\theta(l)}(g)+$ 1 for $g$ (atom for $\left.g^{p_{0}(g) \cdots p_{\theta(l)}(g)}\right)$. Besides this, $I$ is closed, since it is a continuous image of the compact set $J_{\theta(l)}$. The change of coordinates $u_{x, g}:[-1,1] \times I \rightarrow W\left(W=u_{x, g}([-1,1] \times I)\right)$ gives rise, by an affine map $\gamma: I \rightarrow[-1,1]$. to a change of coordinates $\tau:[-1,1]^{2} \rightarrow W$ defined by

$$
\tau\left(x_{1}, x_{2}\right)=u_{x, g}\left(x_{1}, \gamma^{-1}(x)\right) .
$$

By definition, for $y \in[-1,1]$ the leaf $\tau([-1,1] \times\{y\}) \subset W$ intersects $J_{\theta(l)}$ in at least one point. Let $z$ be one point of the intersection $J_{\theta(l)} \cap \tau([-1,1] \times\{y\})$. We have from 9 that $g^{p_{0}(g) \cdots p_{\theta(l)}(g)}(z) \in J_{\theta(l)} \subset W$. This means that $g^{p_{0}(g) \cdots p_{\theta(l)}(g)}(W) \subset W$. Therefore we have:

Lemma 3.19. For any accumulation point $g$ of the sequence of renormalized maps $\mathcal{R}_{n}(f)$ we can find an atom $J$ of the $~^{\text {th }}$ generation and a disk $W$ containing $J$ satisfying the following properties:

(1) Denoting by $q$ the returning time of $J$ into itself (by the map $g$ ) we have that $g^{q}(W) \subset$ $W$.

(2) Under a $C^{k}$ change of coordinates $\tau:[-1,1]^{2} \rightarrow W$, the map $H=\tau^{-1} \circ g^{q} \circ \tau$ reads

$$
H\left(x_{1}, x_{2}\right)=\left(H_{1}\left(0, x_{2}\right), H_{2}\left(0, x_{2}\right)\right) .
$$

Setting $h(x)=H_{2}(0, x), p_{2}\left(x_{1}, x_{2}\right)=x_{2}$ and $\iota_{2}(x)=(0, x)$, the above lemma implies that both diagrams 


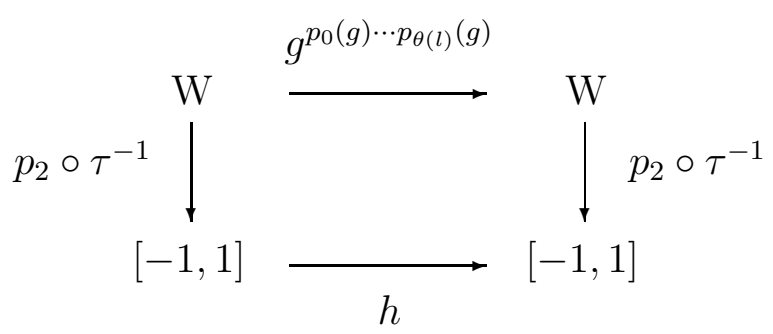

and

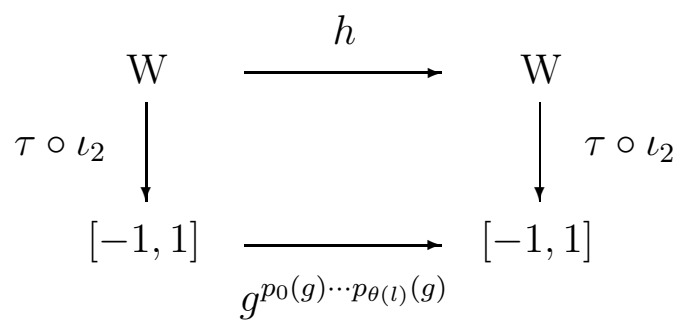

are commutative. Thus, we can recover some of the dynamics of $g^{p_{0}(g) \cdots p_{\theta(l)}(g)}$ in $W$, by the dynamics of $h$. In particular a nested sequence of atoms $\left(K_{m}\right)_{m>0}$ of $g$ of generation $p_{0}(g) \cdots p_{\theta(l)}(g)+m$ in $W$ gives rise to a cascade for $h$ defined by $\overline{\mathcal{D}}_{m}(h)=p_{2} \circ \tau\left(K_{m}\right)$ Therefore $h$ is infinitely renormalizable and the atoms of generation $m$ of $h$ are images by $p_{2} \circ \tau$ of atoms of generation $\theta(l)(g)+m$ of $g$ in $W$. The map $h$ will be called a reduction map. Although the atom $K_{m}$ is not necessarily an embedded disks (g is a degenerate map), we can extend it to an embedded disk $\hat{K}_{m}$ by setting

$$
\hat{K}_{m}=\tau^{-1}\left([-1,1] \times p_{2} \circ \tau\left(K_{m}\right)\right)
$$

With respect to this cascade we have that $\left.g^{p_{0}(g) \cdots p_{\theta(l)}(g)}\right|_{W}$ is infinitely renormalizable.

We begin this section giving a precise meaning to the concept of reduction maps.

Definition 3.20. let $f$ be an infinitely renormalizable $C^{k}$ map of the two disk. We say that a $C^{k}$ map $h:[-1,1] \rightarrow[-1,1]$ is a reduction map for $f$ if we can find another map $h_{1}:[-1,1] \rightarrow[-1,1]$ and an $\left(p_{m}\right)_{m \geq 0}$-infinitely renormalizable map $g: \mathbb{D}^{2} \rightarrow \mathbb{D}^{2}$, satisfying the following conditions:

(1) There is a sequence of integers $\Phi(n)_{n \geq 0}$ such that

$$
\lim _{n \rightarrow \infty} \mathcal{R}_{\Phi(n)}(f) \stackrel{C^{k}}{=} g
$$

(2) There exists an embedded disk $W \subset \mathbb{D}^{2}$ containing an atom $J$ of the $l^{\text {th }}$ generation for $g$ such that $g^{q_{l}}(W) \subset W$, where $q_{l}=p_{0} \cdots p_{l-1}$ is the returning time of $J$ to itself,

(3) Under a $C^{k}$ change of coordinates $\tau:[-1,1]^{2} \rightarrow W, \tau^{-1} \circ g^{q_{l}} \circ \tau$ reads:

$$
\tau^{-1} \circ g^{q_{l}} \circ \tau\left(x_{1}, x_{2}\right)=\left(h_{1}\left(x_{2}\right), h\left(x_{2}\right)\right)
$$


Remark 3.21. Let $\left(K_{m}\right)_{m>0}$ be a nested sequence of atoms in $W$ for the map $g$, with $K_{m}$ of generation $l+m$, involved in the above definition. Then $h$ and $\left.g^{q_{l}}\right|_{W}$ are $\left(p_{m+l}\right)_{m \geq 0^{-}}$ infinitely renormalizable. The sequence

$$
\left(p_{2} \circ \tau\left(K_{m}\right)\right)_{m \geq 0}
$$

is a cascade for $h$ and

$$
\left(\tau^{-1}\left([-1,1] \times p_{2} \circ \tau\left(K_{m}\right)\right)_{m \geq 0}\right.
$$

is a cascade for $\left.g^{q_{l}}\right|_{W}$.

Remark 3.22. For any atom $A$ of $h$, the returning map $h_{\mid A}^{q}: A \rightarrow A$ is also a reduction map for $f$.

Theorem 3.23. Let $1 \leq k \leq \infty$ and $f$ be an infinitely renormalizable $C^{k}$ map of the two disk which contracts the area and satisfies the bounded geometry hypothesis. Then $f$ admits a reduction map.

Proof. Thanks to Corollary [3.6. we find an $C^{k}$-accumulation $g_{0}$ of the sequence of renormalized maps $\left(\mathcal{R}_{n}(f)\right)_{n}$ which is, by Lemma 3.8, an infinitely renormalizable map. Lemma 3.19 applied to $g_{0}$ gives immediately the result by setting $h(x)=H_{2}(0, x)$ as the reduction map.

By the previous theorem, reduction maps always exist provided that $f$ contracts the area and satisfies the bounded geometry hypothesis. We are going now to establish two results on the properties of reduction maps for an infinitely renormalizable maps $f$.

Theorem 3.24. Let $f$ be an $\left(a_{n}\right)_{n \geq 0}$-infinitely renormalizable map satisfying the hypothesis of Theorem 3.23 and let $p_{0}$ be any given accumulation point of the sequence $\left(a_{n}\right)_{n \geq 0}$. Then $f$ admits a reduction map which possesses a periodic point with period $p_{0}$.

Proof. : Let $g_{0}$ be the accumulation point of the sequence of renormalized maps $\mathcal{R}_{n}(f)$ given by Lemma [3.6. Choose a sequence $\Phi_{(n)}$ of integers such that $a_{\Phi(n)}=p_{0}$, and $\lim _{n \rightarrow \infty} \mathcal{R}_{\Phi(n)}(f)=g_{0}$. By Lemma 3.7 we know that $g_{0}$ is renormalizable. More precisely. $\mathcal{R}\left(g_{0}\right)=\zeta_{0}^{-1} \circ g_{0}^{p_{0}} \circ \zeta_{0}$ for an embedding $\zeta_{0}$ which is an accumulation point of $\xi_{\left.\Phi_{(} n\right)}(f)$. Since $g_{0}^{p_{0}}\left(\mathcal{D}_{0}\right) \subset \mathcal{D}_{0}$ for $\mathcal{D}_{0}=\zeta_{0}\left(\mathbb{D}^{2}\right)$, it follows from the Brouwer's Fixed Point Theorem that there is a point $y_{0} \in \mathcal{D}_{0}$ such that $g_{0}^{p_{0}}\left(y_{0}\right)=y_{0}$. Thanks to the bounded geometry hypothesis of $f$, we get from Lemma 3.13 that $g_{0}$ also satisfies the bounded geometry properties. In particular, since their distance is positive, the atoms of the first generation are disjoint. This implies that $y_{0}$ is a periodic orbit with period $p_{0}$ for $g_{0}$.

Consider now the sequence $\left(g_{-l}\right)_{l \geq 1}$ given by Lemma 3.12. Since $\mathcal{R}_{l}\left(g_{-l}\right)=g_{0}$, the existence of a periodic orbit $y_{0}$ for $g_{0}$ implies that in any atom of generation $l$ for the map $g_{-l}$, there is a periodic point of period $p_{0}$ for the map $g_{-l}^{q_{l}}$, where $q_{l}$ denotes the returning time of any atom of generation $l$ of $g_{-l}$.

For every $l \geq 1$, Lemma 3.15 gives a point $x_{l}$ belonging to an atom $J_{l}$ of the $l^{\text {th }}$ generation of $g_{-l}$ for which we have that $\left\|D g_{-l}\left(x_{-l}\right)\right\| \geq 1$. From Theorem 3.4 the sequence $g_{-l}$ admits a converging subsequence in $\mathcal{U}^{k}$. Denote by $(\theta(l))_{l \geq 1}$ a sequence of integers such that $g_{-\theta(l)}$ is convergent in $\mathcal{U}^{k}$ and $x_{\theta(l)}$ also converges to a point $x \in \mathbb{D}^{2}$. Then there exists $l_{0}$ such 
that, for $l \geq l_{0},\left\|D g_{-\theta(l)}(y)\right\| \geq 1 / 2$ for every $y$ in an open neighbourhood $V_{x}$ of $x$. This means, by Theorem 3.14 that $\operatorname{dim}\left(\operatorname{ker}\left(D g_{-\theta(l)}\right)\right)=1$ for every $y \in V_{x}$ and $l \geq l_{0}$.

From now on, we will follow the same kind of strategy as we have done to prove Lemma 3.19

Thanks to Lemma 3.16 we obtain for $l \geq l_{0}$, an embedding $u_{x, g_{-\theta(l)}}:[-1,1]^{2} \rightarrow V_{x}$ such that by setting $g_{\theta(l)}=u_{x, g_{-\theta(l)}^{-1}}^{-} \circ g_{-\theta(l)}^{q_{l}} \circ u_{x, g_{-\theta(l)}}$ we have that

$$
g_{\theta(l)}\left(x_{1}, x_{2}\right)=\left(g_{1, \theta(l)}\left(0, x_{2}\right), g_{2, \theta(l)}\left(0, x_{2}\right)\right) .
$$

Attending to Remark 3.17 we have that for some $\epsilon>0$ and every $l \geq 1, u_{x, g_{-\theta(l)}}\left([-1,1]^{2}\right) \supset$ $B_{\epsilon}(x)$. Applying Lemma 3.13 to the map $g_{-\theta(l)}$ it follows that the atom $J_{\theta(l)}$ satisfies

$$
\left|J_{\theta(l)}\right|<b_{f}^{\theta(l)-1}\left|\mathbb{D}^{2}\right| .
$$

Thus, for $l$ sufficiently large, $J_{\theta(l)} \subset B_{\epsilon}(x)$. Fix an integer $l \geq l_{0}$ such that we have $J_{\theta(l)} \subset B_{\epsilon}(x)$.

Now, set $W=u_{x, g}([-1,1] \times I)$, where $I$ is the projection in the second coordinate of $u_{x, g_{-\theta(l)}}^{-1}\left(J_{\theta(l)}\right)$. By an affine map that carries $I$ to $[-1,1]$ we get from $u_{x, g_{-\theta(l)}}([-1,1] \times I) \rightarrow$ $W$ a change of coordinates $\tau:[-1,1]^{2} \rightarrow W$. It is straightforward to verify that $W \supset J_{\theta(l)}$ and $g_{-\theta(l)}^{q_{\theta(l)}}(W) \subset W$. If we set $g=g_{-\theta(l)}, J=J_{\theta(l)}, H=\tau^{-1} \circ g_{-\theta(l)}^{q_{\theta(l)}} \circ \tau$ we have that

$$
\lim _{n \rightarrow \infty} \mathcal{R}_{\phi(n)}(f) \stackrel{C^{k}}{=} g
$$

and

$$
H\left(x_{1}, x_{2}\right)=\left(H_{1}\left(0, x_{2}\right), H\left(0, x_{2}\right)\right) .
$$

Since the returning time of $J=J_{\theta(l)}$ into itself is $q_{\theta(l)}$, we have that the map $h(x)=H_{2}(0, x)$ is a reduction map for $f$. Because of $g_{\theta(l)}^{q_{\theta(l)}}$ has a periodic orbit of period $p_{0}$ in $J_{\theta(l)}$, we have that $h$ has a periodic orbit of period $p_{0}$.

Theorem 3.25. Let $f$ be an $C^{\infty}$ infinitely renormalizable map of the two disk which contracts the area and satisfies the bounded geometry hypothesis. Then $f$ admits a reduction map $h$ that is multimodal.

Proof. From Lemma 3.25 we get that $f$ admits a $C^{\infty}$ reduction map $h$. From Remark 3.21 we know that $h$ is infinitely renormalizable. Remark 3.22 ensures that any renormalized map of $h$ is also a reduction map for $f$. Thus, to prove the result it suffices to show that there is some $n>0$ such that $h$ has a finite number of critical points in each atom of the $n^{\text {th }}$ generation. We prove this by contradiction.

Suppose that for all $n \geq 1, h$ has in each generation $n$, an atom $I_{n}$ with infinitely many critical points. Denote by $z_{n} \in I_{n}$ an accumulation point of critical points of $h$. In these conditions, $h^{\prime}\left(z_{n}\right)=0$. Moreover, since $z_{n}$ is an accumulation point of infinitely many critical points and $h$ is $C^{\infty}, z_{n}$ is a flat point, i.e. $h^{(k)}\left(z_{n}\right)=0$ for all $k \geq 1$. From the bounded geometry of $g$ and attending to the fact that $p_{2} \circ \tau$ is a Lipschitz function $(\tau$ is 
the change of variables that appears in the Definition [3.20) any atom of generation $n$ has a diameter $d_{n}$ within the range

$$
\begin{aligned}
\frac{1}{C} a_{f}^{p_{0}(g) \cdots p_{\theta(l)}(g)+n} \cdot\left|\mathbb{D}^{2}\right| & \leq d_{n} \leq C \cdot b_{f}^{p_{0}(g) \cdots p_{\theta(l)}(g)+n} \cdot\left|\mathbb{D}^{2}\right| \\
\frac{1}{C_{1}} \cdot a_{f}^{n} & \leq d_{n} \leq C_{1} \cdot b_{f}^{n}
\end{aligned}
$$

where $C_{1}$ is a constant that does not depend on $n$.

By Taylor's expansion, for any $r \geq 1$ there exists $\delta(r)>0$ such that, for any interval $J$ in $I$ with diameter less than $\delta(r)$, we have

$$
|h(J)| \leq \sum_{k=1}^{r-1}\left|h^{(k)}(z)\right||J|^{k}+\|h\|_{r}|I|^{r},
$$

where $z$ is a point in $J$. So, for an integer $N=N(r)$ sufficiently large so that $C_{1} \cdot b_{f}^{n}<\delta(r)$, for $n \geq N$, we obtain choosing $z=z_{n}$, that the image of $I_{n}$ satisfies

$$
\left|h\left(I_{n}\right)\right| \leq C_{2}(r) \cdot b_{f}^{r n} .
$$

Since $h\left(I_{n}\right)$ is another atom of generation $n$, if we take an $r$ such that $b_{f}^{r}<a_{f}$, we get for $n$ sufficiently large a contradiction between 11 and [10.

Remark 3.26. From its proof we can see that it is possible to replace in the previous Theorem the hypothesis on the $C^{\infty}$ differentiability of $f$ by the weaker condition of $f$ being $C^{k}$ for an $k$ satisfying $b_{f}^{k}<a_{f}$.

3.3. Proof of Theorem. Since $f$ is a $(2)_{n \geq 0}$-infinitely renormalizable $C^{\infty}$ map that satisfies the bounded geometry property, Theorem 3.25 says that $f$ has a reduction map $h:[-1,1] \rightarrow[-1,1]$ which is multimodal. The fact that $h$ is a reduction map means that there is another map $h_{1}:[-1,1] \rightarrow[-1,1]$ and an infinitely renormalizable map $g: \mathbb{D}^{2} \rightarrow \mathbb{D}^{2}$, satisfying the following conditions:

(1) There is a sequence of integers $\Phi(n)_{n \geq 0}$ such that

$$
\lim _{n \rightarrow \infty} \mathcal{R}_{\Phi(n)}(f) \stackrel{C^{\infty}}{=} g .
$$

(2) There exists an embedded disk $W \subset \mathbb{D}^{2}$ containing an atom $J$ of the $l^{\text {th }}$ generation for $g$ such that $g^{q_{l}}(W) \subset W$, where $q_{l}$ is the returning time of $J$ into itself,

(3) Under a $C^{\infty}$ change of coordinates $\tau:[-1,1]^{2} \rightarrow W, \tau^{-1} \circ g^{q_{l}}(W) \circ \tau$ reads:

$$
\tau^{-1} \circ g^{q_{l}}(W) \circ \tau\left(x_{1}, x_{2}\right)=\left(h_{1}\left(x_{2}\right), h\left(x_{2}\right)\right) .
$$

We notice that since $g$ is an accumulation point of the sequence of renormalized maps $\mathcal{R}_{n}(f)$, we have by Theorem 3.8 that $g$ is $(2)_{m \geq 0}$ infinitely renormalizable and $q_{l}=2^{l}$.

We extend the map $\tau$ to a homeomorphism $[-2,2]^{2} \rightarrow \mathbb{D}^{2}$, and continue to denote by $\tau$ this extension.

Let $\left(J_{n}\right)_{n \geq 0}$ be the sequence of disks, where $J_{n}$ is the atom of generation $l$ of $\mathcal{R}_{\phi(n)}$ such that $J_{n}$ converges in the Hausdorff topology to $J$. The cascade of periodic orbits $\left(O_{m}(f)\right)_{m>1}$ induces a cascade of periodic orbits $\left(O\left(\mathcal{R}_{k}(f)\right)\right)_{m \geq 1}$ for every renormalized map $\mathcal{R}_{k}(f)$. 
By 4 the signature $s\left(\left(O_{m}(f)\right)_{m \geq 0}\right)$ is an alternating sequence if and only if the signature $s\left(\left(O\left(\mathcal{R}_{k}\right)\right)_{m \geq 1}\right)$ for any renormalized map $\mathcal{R}_{k}(f)$ alternates. Moreover, the map $\mathcal{R}_{\phi(n)}^{2^{l}}$ : $J_{n} \rightarrow J_{n}$ is conjugated to $\mathcal{R}_{\phi(n)+2^{l}}$, and thus the corresponding signature is an alternating sequence if and only if the signature of $f$ is an alternating sequence.

To prove the Theorem 3.1 it suffices to find an integer $n$ such that the signature of the induced cascade of periodic orbits of $\mathcal{R}_{\phi(n)}^{2^{l}}(f)$ in $J_{n}$ is an alternating sequence.

For every integer $n \geq 1$, we define:

$$
\begin{aligned}
H_{n} & =\tau^{-1} \circ \mathcal{R}_{\phi(n)}^{2^{l}}(f) \circ \tau \\
A_{n} & =\tau^{-1}\left(J_{n}\right) .
\end{aligned}
$$

In $A_{n}$, the map $H_{n}$ has a cascade of periodic orbits $\left(O_{n, m}\right)_{m \geq 0}$ that is the conjugated cascade of periodic orbits of $\mathcal{R}_{\phi(n)}^{2^{l}}$ in $J_{n}$. Thus, in order to prove Theorem 3.1 we are going to find an integer $n_{0}$ for which the signature of $\left(O_{n_{0}, m}\right)_{m \geq 0}$ is an alternating sequence.

We set also $H=\tau^{-1} \circ g^{2^{l}} \circ \tau$. For $m$ fixed, the sequence of periodic orbits of period $2^{m}$, $\left(O_{n, m}\right)_{n \geq 1}$ converges as $n \rightarrow \infty$ in the Hausdorff topology to an orbit $O_{m}$ of period $2^{m}$ for $H$, contained in $[-1,1]^{2}$. The projection of $O_{m}$ by $p_{2}\left(x_{1}, x_{2}\right)=x_{2}$ is a periodic orbit $O_{m}^{\prime}$ for $h$. The sequence $\left(O_{m}^{\prime}\right)_{m \geq 0}$ is a period doubling cascade for $h$.

For each $H_{n}$, we can choose an isotopy $\left(H_{n, t}\right)_{t \in[0,1]}$, joining the identity $H_{n, 0}$ to $H_{n}=H_{n, 1}$, in order that the sequence of isotopies is also convergent in the $C^{0}$-topology. It follows that for each $m \geq 1$, there exists $N(m)$ such that $l_{m}\left(H_{n}\right)$ is constant for $n \geq N(m)$. This allows us to extend the linking number $l_{m}$ to the degenerate map $H$ by setting

$$
l_{m}(H)=\lim _{n \rightarrow \infty} l_{m}\left(H_{n}\right) \text {. }
$$

We call $\left(\frac{l_{m}(H)}{2^{m}}\right)_{m}$ the signature of the sequence of periodic orbits $\left(O_{m}\right)_{m}$.

For an embedding $F$ of the disk, and two distinct points $x, y \in \mathbb{D}^{2}$, denote by $\omega_{F}(x, y)$ the algebraic number of half turns of the vector

$$
\left(F_{t}(x)-F_{t}(y)\right) /\left\|F_{t}(x)-F_{t}(y)\right\|,
$$

where by half turns we mean the (algebraic) crossings of the horizontal direction and $\left(F_{t}\right)_{t \in[01]}$ is an isotopy between $F$ and the identity as before.

Since the isotopies $\left(H_{n, t}\right)_{t \in[0,1]}$ are convergent as $n \rightarrow \infty$, we have that for any sequence $\left(x_{n}, y_{n}\right)$ converging to $(x, y)$, there exists $N=N(x, y)$ such that $n \geq N$ implies that $\omega_{H_{n}}\left(x_{n}, y_{n}\right)$ is constant. This allows us to define

$$
\omega_{H}(x, y)=\lim _{n \rightarrow \infty} \omega_{H_{n}}\left(x_{n}, y_{n}\right) .
$$

With this definition, we can write

$$
l_{m}(H)=\sum_{i=0}^{2^{m}-1} w_{H}\left(H^{i}(x), H^{i}(y)\right),
$$

where $x$ is a periodic orbit of period $2^{m}$ which is the parent of the $2^{m+1}$-periodic orbit $y$. 
Now we recall that Theorem 3.25 ensures that the reduction map $h$ is multimodal. Let $I_{1}, \ldots, I_{q}$ the intervals of monotonicity of $h$. The monotonicity of $h$ in each $I_{k}$, implies that $\omega_{H}$ is constant in $\left([-1,1] \times I_{k}\right)^{2}$. We will denote by $M_{k}$ this constant.

Lemma 3.27. We have that

$$
l_{m}(H)=\sum_{k=0}^{q} 2 M_{k} \Phi(k, m)
$$

where $\Phi(k, m)$ denotes de number of points in $O_{m}$ which belong to $[-1,1] \times I_{k}$, or equivalently, the number of points of $O_{m}^{\prime}$ in $I_{k}$.

Proof. Pick a point $x$ of $O_{m}$ in an atom of generation $n$ and a point $y$ in the same atom belonging to $O_{m+1}$. Set $x_{i}=H^{i}(x)$ and $y_{i}=H^{i}(y)$, and denote $p_{2}(t)$ by $t^{\prime}$. We know that $x_{i}^{\prime}$ is always between (its two sons) $y_{i}$ and $y_{i+2^{m}}$. This implies that

$$
w_{H}\left(x_{i}, y_{i}\right)=w_{H}\left(x_{i}, y_{i+2^{m}}\right) .
$$

From 12 it follows that

$$
\begin{aligned}
l_{m}(H) & =\sum_{i=0}^{2^{m+1}-1} w_{H}\left(x_{i}, y_{i}\right) \\
& =\sum_{i=0}^{2^{m+1}-1} w_{H}\left(x_{i}, z_{i}\right),
\end{aligned}
$$

where $z_{i}=y_{i}$ if $x_{i}^{\prime}, y_{i}^{\prime}$ belong to the same $I_{k}$, or $z_{i}=y_{i+2^{m}}$ otherwise. This means that if $x_{i}^{\prime}=x_{i+2^{m}}^{\prime} \in I_{k}$ then $w_{H}\left(x_{i}, z_{i}\right)=w_{H}\left(x_{i+2^{m}}, z_{i+2^{m}}\right)=M_{k}$. Therefore,

$$
\begin{aligned}
l_{m}(H) & =\sum_{i=0}^{2^{m+1}-1} w_{H}\left(x_{i}, z_{i}\right) \\
& =2 \sum_{i=0}^{2^{m}-1} w_{H}\left(x_{i}, z_{i}\right) \\
& =2 \sum_{k=0}^{q} M_{k} \Phi(k, m) .
\end{aligned}
$$

From Proposition 3.3 we know that for all $m \geq 0$ and for all $k \in\{0, \ldots, q\}$

$$
\Phi(k, m+1)=2 \Phi(k, m)+r(k, m)
$$

where for all $p_{2} \geq p_{1} \geq 0$,

$$
\left|\sum_{j=p_{1}}^{p_{2}} r(k, j)\right| \leq 2 .
$$


This together with the previous lemma leads to:

$$
l_{m+1}(H)-2 l_{m}(H)=\sum_{k=1}^{q} 2 M_{k} r(k, m) .
$$

Therefore, by setting $M=\max _{k}\left|M_{k}\right|$ we have for arbitrary $p_{2} \geq p_{1} \geq 0$,

$$
\begin{aligned}
\left|\sum_{m=p_{1}}^{p_{2}} l_{m+1}(H)-2 l_{m}(H)\right| & \leq \sum_{k=1}^{q}\left|2 M_{k}\left(\sum_{m=p_{1}}^{p_{2}} r(k, m)\right)\right| \\
& \leq 4 q M
\end{aligned}
$$

Thus, since $l_{m+1}(H)-2 l_{m}(H)$ are integers, $l_{m+1}(H)-2 l_{m}(H)$ has an infinity of positive and an infinity of negative values.

Then, for an arbitrary integer $N$ we choose $m_{0}$ and $m_{1}$ in order to have $l_{m_{0}+1}(H)-$ $2 l_{m_{0}}(H)>0$ and $l_{m_{1}+1}(H)-2 l_{m_{1}}(H)<0$. But since $l_{m}(H)=\lim _{n \rightarrow \infty} l_{m}\left(H_{n}\right)$, there exists $n_{0}$, such that

$$
\begin{aligned}
l_{m_{0}+1}\left(H_{n_{0}}\right) & =l_{m_{0}+1}(H) \\
l_{m_{0}}\left(H_{n_{0}}\right) & =l_{m_{0}}(H) \\
l_{m_{1}+1}\left(H_{n_{0}}\right) & =l_{m_{1}+1}(H) \\
l_{m_{1}+1}\left(H_{n_{0}}\right) & =l_{m_{1}+1}(H) .
\end{aligned}
$$

Since $N$ is an arbitrary integer, we have that the signature of $\left(O_{n_{0}, m}\right)_{m \geq 0}$ is an alternating sequence and consequently Theorem 3.1 is proved.

\section{REFERENCES}

[1] J. Birman, "Braids, links and mapping class group," Ann. of Math Stud. 4 Princeton Univ. Press (1984).

[2] R. Bowen and J. Franks, "Invariant circles and the order structure of periodic orbits in monotone twist maps," Topology 26 (1987) 21-35.

[3] P. Boyland and G. Hall, "The periodic points of maps of the disk and the interval," Topology 15 (1976) 337-342.

[4] M. Campanino, H Epstein and D. Ruelle, "On the existence of the Feigenbaum's fixed point," Comm. Math. Phys. 79 (1981) 261-302.

[5] M. Campanino and H Epstein, "On Feigenbaum's functional equation $g \circ g(\lambda x)+\lambda g(x)=0$," Topology 21 (1982) 125-129.

[6] E. Catsigeras, "Cascades of period doubling of stable codimension one," IMPA's thesis, 1994.

[7] E. Catsigeras, J-M Gambaudo and F. Moreira, "Infinitely renormalizable diffeomorphisms of the disk at the boundary of chaos," to appear in Proc. of Amer. Math. Soc.

[8] P. Collet, J-P Eckman and H. Koch, "Period doubling bifurcations for families of maps on $\mathbb{R}^{n}$ " J. Stat. Physics, 25 (1980) 1-15.

[9] P. Coullet and C. Tresser, "Itérations d'endomorphismes et groupe de renormalisation," J. Phys. C5 (1978) 25-28.

[10] P. Coullet and C. Tresser and "Itérations d'endomorphismes et groupe de renormalisation," C. R. Acad. Sc. Paris 287A (1978) 577-580. 
[11] O. Courcelle, "Cascades d'orbites périodiques en dimension 1 et 2:hyperbolicité et renormalisaton", Thesis, Université de Nice-Sophia Antipolis,1996.

[12] J-P. Eckman and P. Wittwer, "A complete proof of the Feigenbaum conjectures," J. Stat. Phys. 46 (1987) 455-475.

[13] M. Feigenbaum, "A $C^{2}$ Kupka-Smale diffeomorphism of the disk with no sorces or sinks," "Dynamical Systems and Turbulence" - Lecture Notes in Mathematics . 898 (1980) 90-98.

[14] J. Franks and L.S, Young, "Quantitative universality for a class of non-linear transformations," J. Stat. Phys. 19 (1978) 25-52.

[15] J-M. Gambaudo, "Boundary of Morse-Smale Surface Diffeomorphisms: An Obstruction to Smoothness," in "Proceedings of the International Conference on Dynamical Systems and Related Topics", Advanced Series in Dynamical systems, vol 9 World Scientific, London (1991) 141-152.

[16] J-M. Gambaudo, S. van Strien and C. Tresser, "There exists a $C^{\infty}$ Kupka-Smale diffeomorphism on $\mathbf{S}^{2}$ with neither sinks nor sources," Nonlinearity 2 (1989) 287-304.

[17] J-M. Gambaudo, S. van Strien and C. Tresser, "The periodic orbit structure of orientattion preserving diffeomorphisms on $\mathbb{D}^{2}$ with topological entropy zero," Ann. Inst. H. Poincaré 49 (1989) 335-346.

[18] J-M. Gambaudo, D. Sullivan and C. Tresser, "Infinite cascades of braids and smooth dynamical systems", Topology 33 (1994) 85-94.

[19] J-M. Gambaudo and C. Tresser, "How horseshoes are created," in " Instabilities and Nonequilibrium Structures III" E. Tirapegui and W. Zeller Eds. (Reidel; Dordrecht/Boston/Lancaster/Tokyo) (1991).

[20] J-M. Gambaudo and C. Tresser, "Self-similar constructions in smooth dynamics. Smoothness and dimension," Commun. Math. Phys. 150 (1992) 45-58.

[21] O.E. Lanford III, "A computer -assisted proof of the Feigenbaunm conjecure," Bull. Amer. Math. Soc. 6 (1982) 427-434.

[22] W. de Melo and S. van Strien, One-Dimensional Dynamics (Ergebnisse der Mathematik und ihrer Grenzgebiete. 3. Folge, Vol. 25) (Spinger-Verlag, Berlin, 1993).

[23] D. Smania, "Complex bounds for multimodal maps: Bounded combinatorics," Nonlinearity. 14 (2001) 1311-1330.

[24] D. Sullivan, "Bounds, Quadratic differentials, and Renormalization Conjectures," in A.M.S. Centennial Publication, Vol. (Providence, RI) (1992). 